\title{
THE TRIANGLE OF OPERATORS, TOPOLOGIES, BORNOLOGIES
}

\author{
NGAI-CHING WONG \\ In memory of my teacher Yau-Chuen Wong (1935.10.2-1994.11.7)
}

\begin{abstract}
This paper discusses two common techniques in functional analysis: the topological method and the bornological method. In terms of Pietsch's operator ideals, we establish the equivalence of the notions of operators, topologies and bornologies. The approaches in the study of locally convex spaces of Grothendieck (via Banach space operators), Randtke (via continuous seminorms) and Hogbe-Nlend (via convex bounded sets) are compared.
\end{abstract}

Contents

1. Introduction

2. Established examples in Hilbert spaces and Banach spaces

2.1. The triangle for Hilbert spaces

2.2. The triangle for Banach spaces

3. Notations and Preliminaries

4. The construction and the commutativity of the triangle

5. LCS's defined by operators. topologies and bornologies

6. Examples and Applications

6.1. Schwartz spaces and co-Schwartz spaces

6.2. Nuclear spaces and co-nuclear spaces

6.3. Permanence properties

6.4. Other applications

Date: May 31, 2005.

2000 Mathematics Subject Classification. 47L20, 46A03, 46A11, 46A17.

Key words and phrases. operator ideals, locally convex spaces, topologies, bornologies, nuclear spaces, Schwartz spaces.

Partially supported by Taiwan National Science Council, NSC 83-0208-M-110-017, 93-2115-M-110-009. 


\section{INTRODUCTION}

How can one describe a linear operator $T$ from a Banach space $E$ into a Banach space $F$ ? The usual way to describe $T$ is to state either the bornological property, via $T U_{E}$, or the topological property, via $T^{-1} U_{F}$, of $T$, where $U_{E}$ (resp. $U_{F}$ ) is the closed unit ball of $E$ (resp. $F)$. However, there are a lot of examples indicating that these two machineries are equivalent. For instance,

- $\quad T$ is bounded (i.e., $T U_{E}$ is a bounded subset of $F$ ) $\Leftrightarrow T$ is continuous (i.e., $T^{-1} U_{F}$ is a 0 -neighborhood of $E$ in the norm topology);

- $\quad T$ is of finite rank (i.e., $T U_{E} \subseteq \operatorname{conv}\left\{y_{1}, y_{2}, \ldots, y_{n}\right\}$ for some $y_{1}, y_{2}, \ldots, y_{n}$ in $F$ ) $\Leftrightarrow T$ is weak-norm continuous (i.e., $T^{-1} U_{F}$ is a 0 -neighborhood of $E$ in the weak topology); and

- $\quad T$ is compact (i.e., $T U_{E}$ is totally bounded in $F$ ) $\Leftrightarrow T$ is continuous in the topology of uniform convergence on norm compact subsets of $E^{\prime}$ (i.e., $T^{-1} U_{F} \supseteq K^{\circ}$, the polar of a norm compact subset $K$ of the dual space $E^{\prime}$ of $E)$.

This is because the unit ball of a normed space simultaneously serves as a neighborhood of zero and a bounded set. It is, however, no longer true in the context of locally convex spaces (LCS's, shortly). Mackey-Arens' Theorem indicates that topologies (families of neighborhoods) and bornologies (families of bounded sets) are in dual pair (see e.g. [17]).

It is a long tradition of classifying special classes of locally convex spaces by families of continuous operators among them. A famous example is, of course, Grothendieck's identification of the class of nuclear locally convex spaces. Other examples are those of Schwartz LCS's, infra-Schwartz LCS's and their "co-spaces". After the great effort of Pietsch [15, it is now well-known that such suitable families of continuous operators are the so-called operator ideals.

There are many ways to utilize Grothendieck's idea. For example, one can define a LCS $X$ to be nuclear (resp. Schwartz, infra-Schwartz) by asking that for each continuous seminorm $p$ on $X$, there is a continuous seminorm $q$ on $X$ with $p \leq q$ such that the canonical map $\widetilde{Q}_{p q}$ from $\tilde{X}_{q}=\widehat{X / q^{-1}(0)}$ into $\tilde{X}_{p}=\widehat{X / p^{-1}(0)}$ is nuclear (resp. precompact, weakly compact), where denotes completion. It amounts to saying that the completion $\widetilde{X}$ of $X$ is a topological projective limit $\lim _{\leftarrow} \widetilde{Q}_{p q} \widetilde{X}_{q}$ of Banach spaces of nuclear type (resp. precompact type, weakly compact type). The converse is also true, see Junek [11, p. 139]. We call such a LCS a Grothendieck 
space of nuclear (resp. precompact, weakly compact) type, or shortly a Groth $(\mathfrak{N})$-space (resp. $\operatorname{Groth}\left(\mathfrak{K}_{p}\right)$-space, $\operatorname{Groth}(\mathfrak{W})$-space), where $\mathfrak{N}\left(\operatorname{resp} . \mathfrak{K}_{\mathrm{p}}, \mathfrak{W}\right)$ is the ideal of all nuclear (resp. precompact, weakly compact) operators between Banach spaces.

As a dual concept, a locally convex space $X$ is said to be a co-Grothendieck space of type $\mathfrak{A}$, or shortly a co-Groth( $(\mathfrak{A})$-space, if for each infracomplete disk $A$ in $X$ there is an infracomplete disk $B$ in $X$ such that $A \subseteq B$ and the canonical map $J_{B A}$ from $X(A)=\bigcup_{\lambda>0} \lambda A$ into $X(B)=\bigcup_{\lambda>0} \lambda B$ belongs to $\mathfrak{A}(X(A), X(B))$. In other words, the convex bornological vector space $X$ equipped with the infracomplete bornology of $X$ is the bornological inductive limit $\underset{\mathrm{lim}}{\longrightarrow} J_{B A} X(A)$ of Banach spaces of type $\mathfrak{A}$. The converse is again true.

Another way to go is to define the ideal topology and the ideal bornology on each LCS associated to an operator ideal $\mathfrak{A}$ on $L C S$ 's. A continuous seminorm $p$ on a LCS $X$ is said to be an $\mathfrak{A}$-continuous seminorm if the canonical map $\widetilde{Q}_{p}: X \rightarrow \widetilde{X}_{p}$ belongs to the injective hull $\mathfrak{A}^{\text {inj }}$ of $\mathfrak{A}$. The topology on $X$ defined by the family of all such seminorms is called the $\mathfrak{A}$-topology of $X$. Similarly, an absolutely convex bounded set $B$ in $X$ is said to be $\mathfrak{A}$-bounded if the canonical map $J_{B}$ from $X(B)=\bigcup_{\lambda>0} \lambda B$ into $X$ belongs to the bornologically surjective hull $\mathfrak{A}^{\text {bsur }}$ of $\mathfrak{A}$. The bornology on $X$ defined by the family of all such bounded sets is called the $\mathfrak{A}$-bornology of $X$. A LCS $X$ is said to be $\mathfrak{A}$-topological (resp. $\mathfrak{A}$-bornological) if the topology (resp. bornology) of $X$ coincides with the $\mathfrak{A}$-topology (resp. $\mathfrak{A}$-bornology).

In [26] we show that Grothendieck spaces are essentially a kind of $\mathfrak{A}$-spaces. Thus these two different approaches coincide. In this paper, we will develop the duality theory of $\mathfrak{A}$-topological spaces and $\mathfrak{A}$-bornological spaces. Basically, one may expect that a locally convex space $X$ is $\mathfrak{A}$-topological (resp. $\mathfrak{A}$-bornological) if and only if its strong dual $X_{\beta}^{\prime}$ is $\mathfrak{A}$-bornological (resp. $\mathfrak{A}-$ topological). One can discover the same is true for Grothendieck spaces and co-Grothendieck spaces by observing the duality of topology and bornology and the duality of projective limits and inductive limits (see, e.g., [8, 11]).

The following commutative diagram summaries our works. 


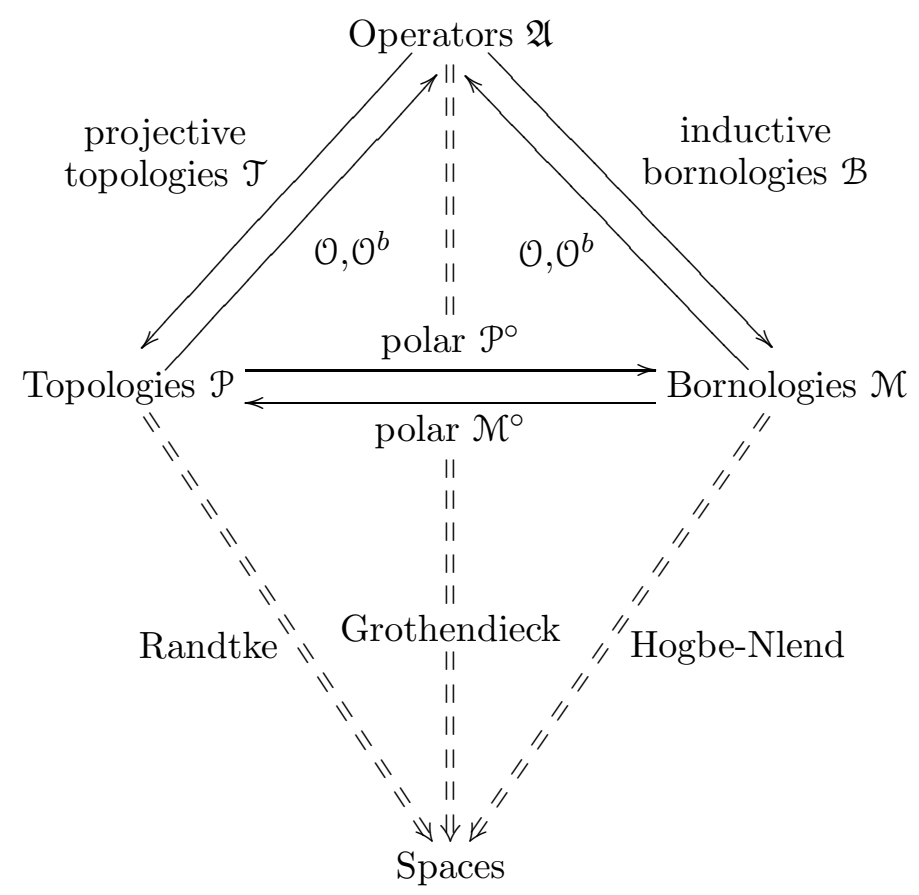

The theory of operator ideal is founded by Pietsch [15] and originated from the works of Grothendieck 44 and Schatten [18. See also [13, 8, 11, 2] for more information. The idea of generating topologies and generating bornologies are due to Stephani [20, 21, 22, 23, 24] and Franco and Piñeiro [3] in the context of Banach spaces. The explicit construction (with all arrows shown in the diagram) of the (upper) triangle is given in 32, in which several applications to Banach space theory are demonstrated. When the underlying space is a fixed complex Hilbert space, West implements the triangle in the context of operator algebras [25] and provides several applications with Conradie [1] (see Section [2). In this paper, we shall complete the LCS version of the triangle. As an application, we shall show that in the study of LCS's, the topological machinery of Randtke (via continuous seminorms) [16] or the bornological machinery of Hogbe-Nlend (via convex bounded subsets) [5, 6] is as strong as that of the operator theoretical machinery of Grothendieck (via Banach space operators) (see e.g. [29, 30, 11, 26]).

The author dedicates this paper to his late teacher, Professor Yau-Chuen Wong, who introduced the same concept of $\mathfrak{A}$-topology and $\mathfrak{A}$-bornology through a great number of examples of special LCS's as well as partially ordered locally convex spaces (see, 29, 30]), although he did not employ the Pietsch's language (operator ideals) at his time. Together with [32, 26], the current paper is a continuation of his ideal (see [27]).

\section{Established examples in Hilbert spaces and Banach spaces}


2.1. The triangle for Hilbert spaces. Let $\mathfrak{M}$ be a von Neumann algebra of bounded linear operators on a Hilbert space $H$, and $\mathfrak{A}$ an arbitrary non-zero two-sided ideal of $\mathfrak{M}$.

- A locally convex topology $\mathcal{P}$ of $H$ is called a generating topology if $\mathcal{P}$ consists of norm open sets in $H$ such that all operators in $\mathfrak{M}$ are $\mathcal{P}$-to- $\mathcal{P}$ continuous on $H$, i.e., the pre-images of $\mathcal{P}$-open sets being $\mathcal{P}$-open.

- A convex vector bornology $\mathcal{M}$ of $H$ is called a generating bornology if $\mathcal{M}$ consists of norm bounded subsets of $H$ such that all operators in $\mathfrak{M}$ are $\mathcal{M}$-to- $\mathcal{M}$ bounded, i.e., sending $\mathcal{M}$-bounded sets to $\mathcal{M}$-bounded sets.

- The $\mathfrak{A}$-topology $\mathfrak{T}(\mathfrak{A})$ is the projective topology of $H$ induced by operators in $\mathfrak{A}$, i.e., the weakest locally convex topology $t$ of $H$ such that operators in $\mathfrak{A}$ are $t$-to-norm continuous.

- The $\mathfrak{A}$-bornology $\mathcal{B}(\mathfrak{A})$ is the inductive bornology of $H$ induced by operators in $\mathfrak{A}$, i.e., the smallest convex vector bornology $b$ of $H$ such that operators in $\mathfrak{A}$ are norm-to- $b$ bounded.

- The polar of a subset $A$ in $H$ is

$$
A^{\circ}=\{x \in H:|\langle a, x\rangle| \leq 1, \forall a \in A\} .
$$

Remark that the ideal $\mathfrak{A}$ is

- self-adjoint, i.e., $T \in \mathfrak{A}$ if and only if its Hilbert space adjoint map $T^{*} \in \mathfrak{A}$;

- injective, i.e., $T \in \mathfrak{A}$ whenever $\|T h\| \leq\|S h\|, \forall h \in H$, for any $S$ in $\mathfrak{A}$ and $T$ in $\mathfrak{M}$; and - surjective, i.e., $T \in \mathfrak{A}$ whenever $T U_{H} \subseteq S U_{H}$ for any $S$ in $\mathfrak{A}$ and $T$ in $\mathfrak{M}$.

Theorem 2.1 (West [25]).

(1) (a) The $\mathfrak{A}$-topology $\mathcal{T}(\mathfrak{A})$ is a generating topology.

(b) The $\mathfrak{A}$-bornology $\mathcal{B}(\mathfrak{A})$ is a generating bornology.

(2) (a) The set $\mathcal{O}(\mathcal{P})=\mathfrak{L}\left(H_{\mathcal{P}}, H\right)$ of all $\mathcal{P}$-to-norm continuous linear operators on $H$ is a two-sided ideals of $\mathfrak{M}$.

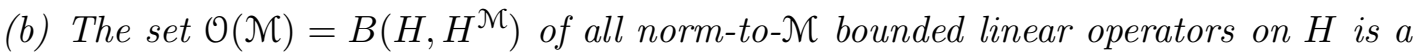
two-sided ideals of $\mathfrak{M}$.

(3) (a) The polar $\mathcal{P}^{\circ}=\left\{B \subseteq H: B^{\circ}\right.$ is a $\mathcal{P}$-neighborhood of 0$\}$ of a generating topology $\mathcal{P}$ is a generating bornology.

(b) The polar $\mathcal{M}^{\circ}=\left\{V \subseteq H: V^{\circ}\right.$ is $\mathcal{M}$-bounded $\}$ of a generating bornology $\mathcal{M}$ is a generating topology. 
(4) The triangle of operators, topologies and bornologies is commutative:

(a) $\mathcal{O}(\mathfrak{T}(\mathfrak{A}))=\mathfrak{A}, \mathcal{O}(\mathcal{B}(\mathfrak{A}))=\mathfrak{A}$.

(b) $\mathcal{T}(\mathfrak{A})^{\circ}=\mathcal{B}(\mathfrak{A}), \mathcal{B}(\mathfrak{A})^{\circ}=\mathfrak{T}(\mathfrak{A})$.

(c) $\mathcal{T}(\mathcal{O}(\mathcal{P}))=\mathcal{P}, \mathcal{B}(\mathcal{O}(\mathcal{M}))=\mathcal{M}$.

2.2. The triangle for Banach spaces. The Banach space version of the "triangle" is known to have many applications (cf. [15, 8, 11]). Let $\mathfrak{A}=\bigcup\{\mathfrak{A}(E, F): E, F$ are Banach spaces $\}$ be an operator ideal on Banach spaces in the sense of Pietsch [15]:

$\left(\mathrm{OI}_{1}\right)$ The components $\mathfrak{A}(E, F)$ of $\mathfrak{A}$ are non-zero subspaces of $\mathfrak{L}(E, F)$.

$\left(\mathrm{OI}_{2}\right) \quad R T S \in \mathfrak{A}\left(E_{0}, F_{0}\right)$ whenever $R \in \mathfrak{L}\left(F, F_{0}\right), T \in \mathfrak{A}(E, F)$ and $S \in \mathfrak{L}\left(E_{0}, E\right)$ for arbitrary Banach spaces $E, E_{0}, F$, and $F_{0}$.

An operator ideal $\mathfrak{A}$ is said to be symmetric if $T \in \mathfrak{A}(E, F)$ ensures its Banach space dual map $T^{\prime} \in \mathfrak{A}\left(F^{\prime}, E^{\prime}\right)$, and completely symmetric if $T \in \mathfrak{A}(E, F) \Leftrightarrow T^{\prime} \in \mathfrak{A}\left(F^{\prime}, E^{\prime}\right)$.

Suppose for each Banach space $E$, we have a locally convex topology $\mathcal{P}(E)$ consisting of norm open subsets of $E$ and a convex vector bornology $\mathcal{M}(E)$ consisting of norm bounded subsets of $E$. We call $\mathcal{P}=\{\mathcal{P}(E): E$ is a Banach space $\}$ a generating topology and $\mathcal{M}=\{\mathcal{M}(E)$ : $E$ is a Banach space $\}$ a generating bornology on Banach spaces, if operators in $\mathfrak{L}(E, F)$ are $\mathcal{P}(E)$-to- $\mathcal{P}(F)$ continuous and $\mathcal{M}(E)$-to- $\mathcal{M}(F)$ bounded for all Banach spaces $E$ and $F$, respectively.

The polar $\mathcal{P}^{\circ}$ of a generating topology $\mathcal{P}$ consists of components

$$
\mathcal{P}^{\circ}(E)=\left\{B \in E: B^{\circ} \text { is } \mathcal{P}\left(E^{\prime}\right) \text {-bounded }\right\} .
$$

Similarly, the polar $\mathcal{M}^{\circ}$ of a generating bornology $\mathcal{M}$ consists of components

$$
\mathcal{M}^{\circ}(E)=\left\{V \in E: V^{\circ} \text { is a } \mathcal{P}\left(E^{\prime}\right) \text {-neighborhood of } 0\right\}
$$

Theorem 2.2 (Stephani [20, 21, 22, 23, 24] and Wong and Wong [32]).

(1) (a) The family of projective topologies $\mathcal{T}(\mathfrak{A})(E)$ of Banach spaces $E$ induced by operators in $\mathfrak{A}(E, \cdot)$ forms a generating topology.

(b) The family of inductive bornologies $\mathcal{B}(\mathfrak{A})(F)$ of Banach spaces $F$ induced by operators in $\mathfrak{A}(\cdot, F)$ forms a generating bornology.

(2) (a) The family of sets $\mathcal{O}(\mathcal{P})(E, F)=\mathfrak{L}\left(E_{\mathcal{P}}, F\right)$ of all $\mathcal{P}(E)$-to-norm continuous linear operators from $E$ into $F$ forms an injective operator ideal. 
THE TRIANGLE OF OPERATORS, TOPOLOGIES, BORNOLOGIES

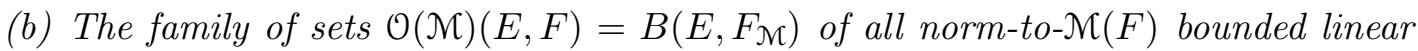
operators from $E$ into $F$ forms a surjective operator ideal.

(3) (a) The polar $\mathcal{P}^{\circ}$ of a generating topology $\mathcal{P}$ is a generating bornology.

(b) The polar $\mathcal{M}^{\circ}$ of a generating bornology $\mathcal{M}$ is a generating topology.

(4) The triangle of operators, topologies and bornologies is almost commutative.

(a) $\mathcal{O}(\mathcal{T}(\mathfrak{A}))=\mathfrak{A}^{\text {inj }}$ and $\mathcal{O}(\mathcal{B}(\mathfrak{A}))=\mathfrak{A}^{\text {sur }}$.

(b) $\mathcal{B}(\mathfrak{A})^{\circ}=\mathfrak{T}(\mathfrak{A})$ if $\mathfrak{A}$ is symmetric, and $\mathcal{T}(\mathfrak{A})^{\circ}=\mathfrak{B}(\mathfrak{A})$ if $\mathfrak{A}$ is completely symmetric.

(c) $\mathcal{T}(\mathcal{O}(\mathcal{P}))=\mathcal{P}, \mathcal{B}(\mathcal{O}(\mathcal{M}))=\mathcal{M}$.

Note that the ideals $\mathfrak{L}$ of all bounded operators, $\mathfrak{F}$ of all bounded operator of finite rank and $\mathfrak{K}$ of all compact operators are all injective, surjective and completely symmetric. These explain the equivalence of topological and bornological approaches for these operators demonstrated at the very beginning of this paper. On the other hand, the ideal $\mathfrak{N}$ of nuclear operators is neither injective, surjective or completely symmetric (cf. [15]).

\section{Notations and Preliminaries}

The classic reference to the theory of operator ideals is, of course, Pietsch [15]. See also Jarchow [8] and Junek [1]. For the theory of locally convex spaces, together with Wong [31], Schaefer [17] is our favorite. Hogbe-Nlend [5] serves as our main source of the theory of bornology.

Throughout this paper, all vector spaces have the same underlying scalar field $\mathbb{K}$. $\mathbb{K}$ is either the field $\mathbb{R}$ of real numbers or the field $\mathbb{C}$ of complex numbers. Locally convex topologies are always Hausdorff, and convex vector bornologies are always separated, i.e., no nonzero subspace is bounded. Operators always refer to linear maps without any topological or bornological assumption. $U_{N}$ always denotes the closed unit ball of a normed space $N$.

A subset $B$ of a LCS $X$ is said to be a disk if $B$ is absolutely convex, i.e., $\alpha B+\beta B \subseteq B$ whenever $|\alpha|+|\beta| \leq 1$. A disk $B$ is said to be a $\sigma$-disk, or absolutely $\sigma$-convex if $\Sigma_{n} \lambda_{n} b_{n}$ converges in $B$ whenever $\sum_{n}\left|\lambda_{n}\right| \leq 1$ and $b_{n} \in B, n=1,2, \ldots$ A bounded disk $B$ is said to be infracomplete (or a Banach disk) if the normed space $X(B)=\bigcup_{\lambda>0} \lambda B$ equipped with the gauge $\gamma_{B}$ of $B$ as its norm is complete, where $\gamma_{B}(x)=\inf \{\lambda>0: x \in \lambda B\}$, for each $x$ in $X(B)$. Any continuous image of a $\sigma$-disk or an infracomplete bounded disk is still a $\sigma$-disk or an infracomplete bounded disk, respectively. A LCS $X$ is said to be infracomplete if the von Neumann bornology $\mathcal{M}_{\mathrm{von}}(X)$, i.e., the original bornology induced by the topology of $X$, has a 
basis consisting of infracomplete subsets of $X$, or equivalently, $\sigma$-disked subsets of $X$. In other words, $\left(X, \mathcal{M}_{\mathrm{von}}(X)\right)$ is a complete convex bornological vector space.

Let $\left\langle X, X^{\prime}\right\rangle$ be a dual pair and $B \subseteq X$. The (absolute) polar $B^{\circ}$ of $B$ in $X^{\prime}$ is defined by

$$
B^{\circ}=\left\{x \in X^{\prime}:|\langle b, x\rangle| \leq 1, \quad \forall b \in B\right\} .
$$

Whenever $A \subseteq X^{\prime}$, denote by $A^{\bullet}$ the polar of $A$ taken in $X_{\beta \beta}^{\prime \prime}$, namely,

$$
A^{\bullet}=\left\{x \in X_{\beta \beta}^{\prime \prime}:|\langle a, x\rangle| \leq 1, \quad \forall a \in A\right\}
$$

where $X_{\beta \beta}^{\prime \prime}$ is the strong bidual of $X$, while $A^{\circ}$ denotes the polar of $A$ taken in $X$ with respect to the dual pair $\left\langle X, X^{\prime}\right\rangle$.

Proposition 3.1 (See, e.g., Wong [31, pp. 224 and 227]). Let $X$ and $Y$ be LCS's and $T \in$ $\mathcal{L}(X, Y)$. We have

(1) $T \in \mathcal{L}\left(X_{\sigma}, Y_{\sigma}\right)$, where $X_{\sigma}, Y_{\sigma}$ denote the LCS's in their weak topologies.

(2) $T \in \mathcal{L}\left(X_{\tau}, Y_{\tau}\right)$, where $X_{\tau}, Y_{\tau}$ denote the LCS's in their Mackey topologies.

(3) $T^{\prime} \in \mathcal{L}\left(Y_{\beta}^{\prime}, X_{\beta}^{\prime}\right)$, where $T^{\prime}$ is the dual map of $T$ and $X_{\beta}^{\prime}$ (resp. $Y_{\beta}^{\prime}$ ) is the strong dual of $X$ (resp. $Y)$.

(4) $(T A)^{\circ}=\left(T^{\prime}\right)^{-1} A^{\circ}$ for all nonempty subset $A$ of $X$.

(5) $\left(T^{\prime} B\right)^{\circ}=T^{-1} B^{\circ}$ for all nonempty subset $B$ of $Y^{\prime}$.

(6) $\left(T^{-1} W\right)^{\circ}=T^{\prime} W^{\circ}$ for all neighborhoods $W$ of 0 in its Mackey topology $\tau\left(Y, Y^{\prime}\right)$.

Let $X$ and $Y$ be LCS's. $J$ in $\mathfrak{L}(X, Y)$ is called a (topological) injection if $J$ is one-to-one and relatively open. $Q$ in $\mathfrak{L}(X, Y)$ is called a (topological) surjection if $Q$ is open (and thus $Q$ induces the topology of $Y) . Q^{1}$ in $\mathfrak{L}(X, Y)$ is called a bornological surjection if $Q^{1}$ is onto and induces the bornology of $Y$ (i.e., for each bounded subset $B$ of $Y$ there is a bounded subset $A$ of $X$ such that $Q^{1} A=B$ ).

An operator ideal $\mathfrak{A}$ on LCS's is said to be

- injective if $J T \in \mathfrak{A}\left(X, Y_{0}\right)$ infers $T \in \mathfrak{A}(X, Y)$, whenever $T \in \mathfrak{L}(X, Y)$ and $J \in \mathfrak{L}\left(Y, Y_{0}\right)$ is an injection for some $\operatorname{LCS} Y_{0}$;

- surjective if $T Q \in \mathfrak{A}\left(X_{0}, Y\right)$ infers $T \in \mathfrak{A}(X, Y)$, whenever $T \in \mathfrak{L}(X, Y)$ and $Q \in$ $\mathfrak{L}\left(X_{0}, X\right)$ is a surjection for some LCS $X_{0}$; and

- bornologically surjective if $T Q^{1} \in \mathfrak{A}\left(X_{0}, Y\right)$ infers $T \in \mathfrak{A}(X, Y)$, whenever $T \in \mathfrak{L}(X, Y)$ and $Q^{1} \in \mathfrak{L}\left(X_{0}, X\right)$ is a bornological surjection for some LCS $X_{0}$. 
The injective hull $\mathfrak{A}^{\text {inj }}$, the surjective hull $\mathfrak{A}^{\text {sur }}$, and the bornologically surjective hull $\mathfrak{A}^{\text {bsur }}$ of $\mathfrak{A}$ is the intersection of all injective, surjective, and bornologically surjective operator ideals containing $\mathfrak{A}$, respectively. Note that for operator ideals on Banach spaces, the notions of surjectivity and bornological surjectivity coincide.

Associate to each normed space $N$ the Banach space $N^{\text {inj }}=l_{\infty}\left(U_{N^{\prime}}\right)$ and the injection $J_{N}$ in $\mathfrak{L}\left(N, N^{\text {inj }}\right)$ defined by $J_{N}(x)=(\langle x, a\rangle)_{a \in U_{N^{\prime}}}$. Similarly, we define $N^{\text {sur }}$ to be the normed space $L_{1}\left(U_{N}\right)=\left\{\left(\lambda_{x}\right) \in \ell_{1}\left(U_{N}\right): \sum_{x \in U_{N}} \lambda_{x} x\right.$ converges in $\left.N\right\}$ and $Q_{N}: N^{\text {sur }} \rightarrow N$ to be the surjection defined by $Q_{N}\left(\left(\lambda_{x}\right)_{x \in U_{N}}\right)=\sum_{x \in U_{N}} \lambda_{x} x$. In case $E$ is a Banach space, it is well-known that $E^{\text {inj }}$ has the extension property and $E^{\text {sur }}$ has the lifting property, cf. [15].

Proposition 3.2 ([15, 3, 28,).

(1) Let $\mathfrak{A}$ be an operator ideal on Banach spaces.

$$
\begin{aligned}
\mathfrak{A}^{\mathrm{inj}}(E, F) & =\left\{R \in \mathfrak{L}(E, F): J_{F} R \in \mathfrak{A}\left(E, F^{\mathrm{inj}}\right)\right\}, \\
\mathfrak{A}^{\text {sur }}(E, F) & =\left\{S \in \mathfrak{L}(E, F): S Q_{E} \in \mathfrak{A}\left(E^{\text {sur }}, F\right)\right\} .
\end{aligned}
$$

(2) Let $\mathfrak{A}$ be an operator ideal on LCS's. We can associate to each LCS Y a LCS $Y^{\infty}$ and an injection $J_{Y}^{\infty}$ from $Y$ into $Y^{\infty}$, and to each LCS X a LCS $X^{1}$ and a bornological surjection $Q_{X}^{1}$ from $X^{1}$ onto $X$ such that

$$
\begin{aligned}
\mathfrak{A}^{\mathrm{inj}}(X, Y) & =\left\{R \in \mathfrak{L}(X, Y): J_{Y}^{\infty} R \in \mathfrak{A}\left(X, Y^{\infty}\right)\right\}, \\
\mathfrak{A}^{\text {bsur }}(X, Y) & =\left\{S \in \mathfrak{L}(X, Y): S Q_{X}^{1} \in \mathfrak{A}\left(X, Y^{1}\right)\right\} .
\end{aligned}
$$

Moreover, we have

$$
\mathfrak{A}^{\text {inj bsur }}=\mathfrak{A}^{\text {bsur inj }} .
$$

In case $N$ is a normed space, $R \in \mathfrak{L}(X, N)$ and $S \in \mathfrak{L}(N, Y)$,

$$
\begin{aligned}
J_{N} R \in \mathfrak{A}\left(X, N^{\mathrm{inj}}\right) & \Longleftrightarrow J_{N}^{\infty} R \in \mathfrak{A}\left(X, N^{\infty}\right), \\
S Q_{N}^{1} \in \mathfrak{A}\left(N^{1}, Y\right) & \Longleftrightarrow S Q_{N} \in \mathfrak{A}\left(N^{\text {sur }}, Y\right) .
\end{aligned}
$$

\section{The COnstruction And the COMmutativity of the triangle}

Let $\mathcal{C}$ be a class of locally convex spaces. Let $X, Y \in \mathcal{C}$. We denote by $\mathfrak{L}^{b}(X, Y), \mathfrak{L}(X, Y)$ and $L^{\times}(X, Y)$ the collection of all operators from $X$ into $Y$ which are bounded (i.e., sending a 0-neighborhood to a bounded set), continuous, and locally bounded (i.e., sending bounded sets to bounded sets), respectively. 
Denote by $\sigma\left(X, X^{\prime}\right)$ the weak topology of $X$ with respect to its dual space $X^{\prime}$, while $\mathcal{P}_{\text {ori }}(X)$ is the original topology of $X$. We employ the notion $\mathcal{M}_{\text {fin }}(Y)$ for the finite dimensional bornology of $Y$ which has a basis consisting of all convex hulls of finite sets. On the other hand, $\mathcal{M}_{\text {von }}(Y)$ is used for the von Neumann bornology of $Y$ which consists of all topologically bounded subsets of $Y$. Ordering of topologies and bornologies are induced by set-theoretical inclusion, as usual. Moreover, we write briefly $X_{\mathcal{P}}$ for a vector space $X$ equipped with a locally convex topology $\mathcal{P}$ and $Y^{\mathcal{M}}$ for a vector space $Y$ equipped with a convex vector bornology $\mathcal{M}$.

We now give the details of the "triangle".

\section{Definition 4.1.}

(1) ("Operators") A family $\mathfrak{A}=\{\mathfrak{A}(X, Y): X, Y \in \mathcal{C}\}$ of algebras of operators associated to each pair of spaces $X$ and $Y$ in $\mathcal{C}$ is called an operator ideal if

$\mathrm{OI}_{1}: \mathfrak{A}(X, Y)$ is a nonzero vector subspace of $\mathfrak{L}(X, Y)$ for all $X, Y$ in $\mathcal{C}$; and

$\mathbf{O I}_{2}: R T S \in \mathfrak{A}\left(X_{0}, Y_{0}\right)$ whenever $R \in \mathfrak{L}\left(Y, Y_{0}\right), T \in \mathfrak{A}(X, Y)$ and $S \in \mathfrak{L}\left(X_{0}, X\right)$ for any $X_{0}, X, Y$ and $Y_{0}$ in $\mathrm{e}$.

(2) ("Topologies") A family $\mathcal{P}=\{\mathcal{P}(X): X \in \mathcal{C}\}$ of locally convex topologies associated to each space $X$ in $\mathcal{C}$ is called a generating topology if

$\mathbf{G T}_{1}: \sigma\left(X, X^{\prime}\right) \subseteq \mathcal{P}(X) \subseteq \mathcal{P}_{\text {ori }}(X)$ for all $X$ in $\mathcal{C}$; and

$\mathbf{G T}_{2}: \mathfrak{L}(X, Y) \subseteq \mathfrak{L}\left(X_{\mathcal{P}}, Y_{\mathcal{P}}\right)$ for all $X$ and $Y$ in $\mathcal{C}$.

(3) ("Bornologies") A family $\mathcal{M}=\{\mathcal{N}(Y): Y \in \mathcal{C}\}$ of convex vector bornologies associated to each space $Y$ in $\mathcal{C}$ is called a generating bornology if

$\mathbf{G B}_{1}: \mathcal{M}_{\text {fin }}(Y) \subseteq \mathcal{M}(Y) \subseteq \mathcal{M}_{\text {von }}(Y)$ for all $Y$ in $\mathcal{C}$; and

$\mathbf{G B}_{2}: \mathfrak{L}(X, Y) \subseteq L^{\times}\left(X^{\mathcal{M}}, Y^{\mathcal{M}}\right)$ for all $X$ and $Y$ in $\mathcal{C}$.

Classical examples of these notions are the ideals $\mathfrak{K}_{p}$ of precompact operators and $\mathfrak{P}$ of abso-

lutely summing operators (see e.g. [15]), the generating systems $\mathcal{P}_{p c}$ of precompact topologies (see e.g. [16]) and $\mathcal{P}_{p n}$ of prenuclear topologies (see e.g. [17 p. 90]), and the generating systems $\mathcal{M}_{p c}$ of precompact bornologies and $\mathcal{M}_{p n}$ of prenuclear bornologies (see e.g. [6]), respectively. An interesting fact about these examples is that we can visualize the notions of "operators", "topologies" and "bornologies" as vertices of a triangle, and they can be transformed to each other by actions represented as linking edges of the triangle.

Definition 4.2. Let $\mathfrak{A}$ be an operator ideal, $\mathcal{P}$ a generating topology and $\mathcal{M}$ a generating bornology on $\mathrm{C}$. 
(1) ("Operators" $\rightarrow$ "Topologies") For each $X_{0}$ in $\mathcal{C}$, the $\mathfrak{A}$-topology of $X_{0}$, denoted by $\mathcal{T}(\mathfrak{A})\left(X_{0}\right)$, is the projective topology of $X_{0}$ with respect to the family

$$
\left\{T \in \mathfrak{A}\left(X_{0}, Y\right): Y \in \mathcal{C}\right\} .
$$

In other words, a seminorm $p$ of $X_{0}$ is $\mathcal{T}(\mathfrak{A})\left(X_{0}\right)$-continuous if and only if there is a $T$ in $\mathfrak{A}\left(X_{0}, Y\right)$ for some $Y$ in $\mathcal{C}$ and a continuous seminorm $q$ of $Y$ such that

$$
p(x) \leq q(T x), \quad \forall x \in X_{0} .
$$

In this case, we call $p$ an $\mathfrak{A}-$ seminorm of $X_{0}$.

(2) ("Operators" $\rightarrow$ "Bornologies") For each $Y_{0}$ in $\mathcal{C}$, the $\mathfrak{A}$-bornology of $Y_{0}$, denoted by $\mathcal{B}(\mathfrak{A})\left(Y_{0}\right)$, is the inductive bornology of $Y_{0}$ with respect to the family

$$
\left\{T \in \mathfrak{A}\left(X, Y_{0}\right): X \in \mathcal{C}\right\} .
$$

In other words, a subset $B$ of $Y_{0}$ is $\mathcal{B}(\mathfrak{A})\left(Y_{0}\right)$-bounded if and only if there is a $T$ in $\mathfrak{A}\left(X, Y_{0}\right)$ for some $X$ in $\mathcal{C}$ and a topologically bounded subset $A$ of $X$ such that

$$
B \subseteq T A .
$$

In this case, we call $B$ an $\mathfrak{A}$-bounded subset of $Y_{0}$.

(3) ("Topologies" $\rightarrow$ "Operators") For $X, Y$ in $\mathcal{C}$, let

$$
\mathcal{O}(\mathcal{P})(X, Y)=\mathfrak{L}\left(X_{\mathcal{P}}, Y\right)
$$

and

$$
\mathcal{O}^{b}(\mathcal{P})(X, Y)=\mathfrak{L}^{b}\left(X_{\mathcal{P}}, Y\right)
$$

be the vector space of all continuous operators from $X$ into $Y$ which is still continuous with respect to the $\mathcal{P}(X)$-topology, and which send a $\mathcal{P}(X)$-neighborhood of zero to a bounded set, respectively.

(4) ("Bornologies" $\rightarrow$ "Operators") For $X, Y$ in $\mathcal{C}$, let

$$
\mathcal{O}(\mathcal{M})(X, Y)=\mathfrak{L}(X, Y) \cap L^{\times}\left(X, Y^{\mathcal{M}}\right)
$$

and

$$
\mathcal{O}^{b}(\mathcal{M})(X, Y)=\mathfrak{L}^{b}\left(X, Y^{\mathcal{M}}\right)
$$

be the vector space of all continuous operators from $X$ into $Y$ which send bounded sets to $\mathcal{M}(Y)$-bounded sets, and which send a neighborhood of zero to an $\mathcal{M}(Y)$-bounded set, respectively.

(5) ("Topologies" $\leftrightarrow$ "Bornologies") For $X, Y$ in $\mathcal{C}$, the $\mathcal{P}^{\circ}(Y)$-bornology of $Y$ (resp. $\mathcal{M}^{\circ}(X)$-topology of $X$ ) is defined to be the bornology (resp. topology) polar to $\mathcal{P}(X)$ (resp. $\mathcal{M}(Y))$. More precisely, 
- a bounded subset $A$ of $Y$ is $\mathcal{P}^{\circ}(Y)$-bounded if and only if its polar $A^{\circ}$ is a $\mathcal{P}\left(Y_{\beta}^{\prime}\right)$ neighborhood of zero; and

- a neighborhood $V$ of zero of $X$ is a $\mathcal{M}^{\circ}(X)$-neighborhood of zero if and only if $V^{\circ}$ is $\mathcal{M}\left(X_{\beta}^{\prime}\right)$-bounded.

Theorem 4.3. Let $\mathfrak{A}$ be an operator ideal, $\mathcal{P}$ a generating topology and $\mathcal{M}$ a generating bornology on $\mathrm{C}$. We have

(1) $\mathcal{T}(\mathfrak{A})=\{\mathfrak{T}(\mathfrak{A})(X): X \in \mathcal{C}\}$ is a generating topology on $\mathcal{C}$.

(2) $\mathcal{B}(\mathfrak{A})=\{\mathcal{B}(\mathfrak{A})(Y): Y \in \mathcal{C}\}$ is a generating bornology on $\mathcal{C}$.

(3) $\mathcal{O}(\mathcal{P})=\{\mathcal{O}(\mathcal{P})(X, Y): X, Y \in \mathcal{C}\}$ is an operator ideal on $\mathcal{C}$.

(4) $\mathcal{O}^{b}(\mathcal{P})=\left\{\mathcal{O}^{b}(\mathcal{P})(X, Y): X, Y \in \mathcal{C}\right\}$ is an operator ideal on $\mathcal{C}$.

(5) $\mathcal{O}(\mathcal{M})=\{\mathcal{O}(\mathcal{M})(X, Y): X, Y \in \mathcal{C}\}$ is an operator ideal on $\mathcal{C}$.

(6) $\mathcal{O}^{b}(\mathcal{M})=\left\{\mathcal{O}^{b}(\mathcal{M})(X, Y): X, Y \in \mathcal{C}\right\}$ is an operator ideal on $\mathcal{C}$.

(7) $\mathcal{P}^{\circ}=\left\{\mathcal{P}^{\circ}(Y): Y \in \mathcal{C}\right\}$ is a generating bornology on $\mathcal{C}$.

(8) $\mathcal{M}^{\circ}=\left\{\mathcal{M}^{\circ}(Y): Y \in \mathcal{C}\right\}$ is a generating topology on $\mathcal{C}$.

Proof. (1)-(6), together with the Banach space version of (7) and (8), are done in [32]. For the locally convex space version of $(7)$, we first note that $\left(\mathrm{GB}_{1}\right)$ follows from $\left(\mathrm{GT}_{1}\right)$ and the bipolar theorem. To check $\left(\mathrm{GB}_{2}\right)$, let $X$ and $Y$ be LCS's and $T \in \mathfrak{L}(X, Y)$. Let $B$ be a $\mathcal{P}^{\circ}(X)$-bounded subset of $X$ and we want to see that $T B$ is $\mathcal{P}^{\circ}(Y)$-bounded in $Y$. Since $B^{\circ}$ is a $\mathcal{P}\left(X_{\beta}^{\prime}\right)$-neighborhood of zero of the strong dual $X_{\beta}^{\prime}$ of $X,(T B)^{\circ}=\left(T^{\prime}\right)^{-1} B^{\circ}$ is a $\mathcal{P}\left(Y_{\beta}^{\prime}\right)^{-}$ neighborhood of zero of $Y_{\beta}^{\prime}$ as a consequence of $\left(\mathrm{GT}_{2}\right)$ and the fact that $T^{\prime} \in \mathfrak{L}\left(Y_{\beta}^{\prime}, X_{\beta}^{\prime}\right)$. Hence, $T B$ is $\mathcal{P}^{\circ}(Y)$-bounded in $Y$, as asserted.

Finally, for (8) we note that $\left(\mathrm{GT}_{1}\right)$ is plain. For $\left(\mathrm{GT}_{2}\right)$, let $X$ and $Y$ be LCS's and $T \in$ $\mathfrak{L}(X, Y)$. Let $V$ be a $\mathcal{M}^{\circ}(Y)$-neighborhood of zero of $Y$ and we want to see that $T^{-1} V$ is an $\mathcal{M}(X)$-neighborhood of zero of $X$. Since $V^{\circ}$ is $\mathcal{M}\left(Y_{\beta}^{\prime}\right)$-bounded in $Y_{\beta}^{\prime},\left(T^{-1} V\right)^{\circ}=T^{\prime} V^{\circ}$ is a $\mathcal{M}\left(X_{\beta}^{\prime}\right)$-bounded subset of $X_{\beta}^{\prime}$, as asserted.

Remark 4.4. A seemingly more general setting is to define for generating topologies $\mathcal{P}$ and $\mathcal{P}_{1}$, and generating bornologies $\mathcal{M}$ and $\mathcal{M}_{1}$ the operator ideals with components $\mathcal{O}\left(\mathcal{P} / \mathcal{P}_{1}\right)(X, Y)=$ $\mathfrak{L}\left(X_{\mathcal{P}}, Y_{\mathcal{P}_{1}}\right), \mathcal{O}\left(\mathcal{M} / \mathcal{M}_{1}\right)(X, Y)=L^{\times}\left(X^{\mathcal{M}}, Y^{\mathcal{M}_{1}}\right) \cap \mathfrak{L}(X, Y)$ and $\mathcal{O}(\mathcal{P} / \mathcal{M})(X, Y)=\mathfrak{L}\left(X_{\mathcal{P}}, Y^{\mathcal{M}}\right)$. However, they will not give rise to new tools to us. In fact, we have $\mathcal{O}\left(\mathcal{P} / \mathcal{P}_{1}\right)=\mathcal{O}\left(\mathcal{P}_{1}\right)^{-1} \circ \mathcal{O}(\mathcal{P})$ [23, $\mathcal{O}\left(\mathcal{M} / \mathcal{M}_{1}\right)=\mathcal{O}(\mathcal{M}) \circ \mathcal{O}\left(\mathcal{M}_{1}\right)^{-1}\left[24\right.$, and $\mathcal{O}(\mathcal{P} / \mathcal{M})=\mathcal{O}^{b}(\mathcal{M}) \circ \mathcal{O}(\mathcal{P})=\mathcal{O}(\mathcal{M}) \circ \mathcal{O}^{b}(\mathcal{P})$. Readers are referred to Pietsch's classic [15] for information regarding quotients and products of operator ideals. 
Let $p$ be a continuous seminorm of a LCS $X$ and $B$ an absolutely convex bounded subset of a LCS $Y$. Denote by $X_{p}$ the normed space $X / p^{-1}(0)$ equipped with norm $\left\|x+p^{-1}(0)\right\|=p(x)$, and by $Y(B)$ the normed space $\bigcup_{\lambda>0} \lambda B$ equipped with norm $r_{B}(x)=\inf \{\lambda>0: x \in \lambda B\}$. Let $\widetilde{X}_{p}$ be the completion of $X_{p}$. Define $Q_{p}: X \longrightarrow X_{p}, \widetilde{Q}_{p}: X \longrightarrow \widetilde{X}_{p}$ and $J_{B}: Y(B) \longrightarrow Y$ to be the canonical maps.

Theorem 4.5 ([26]). Let $\mathfrak{A}$ be an operator ideal on $L C S$ 's. We have

(1) A continuous seminorm $p$ of $X$ is an $\mathfrak{A}$-seminorm if and only if $Q_{p} \in \mathfrak{A}^{\mathrm{inj}}\left(X, X_{p}\right)$ if and only if $\widetilde{Q}_{p} \in \mathfrak{A}^{\operatorname{inj}}\left(X, \widetilde{X}_{p}\right)$.

(2) A bounded disk $B$ of $Y$ is an $\mathfrak{A}$-bounded set if and only if $J_{B} \in \mathfrak{A}^{\text {bsur }}(Y(B), Y)$. Whenever $\mathfrak{A}$ is surjective, we can replace $\mathfrak{A}^{\text {bsur }}$ by $\mathfrak{A}^{\text {sur }}$.

For operator ideals $\mathfrak{A}$ on Banach spaces, Stephani [20, 22] achieved that $\mathcal{O}(\mathfrak{T}(\mathfrak{A}))=\mathfrak{A}^{\text {inj }}$ and $\mathcal{O}(\mathcal{B}(\mathfrak{A}))=\mathfrak{A}^{\text {sur }}$. However, we have two constructions $\mathcal{O}$ and $\mathcal{O}^{b}$ in the context of LCS's. Unlike the Banach space version, they give rise to different ideals. For example, let $\mathcal{M}_{p c}$ be the generating system of precompact bornologies (i.e., the bornologies determined by totally bounded convex sets). Then $\mathfrak{K}_{p}=\mathcal{O}^{b}\left(\mathcal{M}_{p c}\right)$ is the ideal of precompact operators (i.e., those sending a neighborhood of zero to a totally bounded set) and $\mathfrak{K}_{p}^{l o c}=\mathcal{O}\left(\mathcal{M}_{p c}\right)$ is the ideal of locally precompact operators (i.e., those sending bounded sets to totally bounded sets). Randtke [16] indicated that $\mathfrak{K}_{p}(X, Y)=\mathfrak{K}_{p}^{l o c}(X, Y)$ holds for all LCS $Y$ if and only if $X$ is a Schwartz space. On the other hand, it is straightforward to make the following observation.

Proposition 4.6. For a generating topology $\mathcal{P}$ and a generating bornology $\mathcal{M}$ on $L C S$ 's, $\mathcal{O}(\mathcal{P})$ and $\mathcal{O}^{b}(\mathcal{P})$ give rise to the same ideal topology, namely

$$
\mathcal{T}(\mathcal{O}(\mathcal{P}))=\mathcal{T}\left(\mathcal{O}^{b}(\mathcal{P})\right)=\mathcal{P}
$$

and $\mathcal{O}(\mathcal{M})$ and $\mathcal{O}^{b}(\mathcal{M})$ give rise to the same ideal bornology, namely

$$
\mathcal{B}(\mathcal{O}(\mathcal{M}))=\mathcal{B}\left(\mathcal{O}^{b}(\mathcal{M})\right)=\mathcal{M}
$$

Moreover, $\mathcal{O}(\mathcal{P})$ and $\mathcal{O}^{b}(\mathcal{P})$ are injective, $\mathcal{O}(\mathcal{M})$ is bornologically surjective and $\mathcal{O}^{b}(\mathcal{M})$ is surjective.

Proposition 4.7. Let $\mathfrak{A}$ be an operator ideal on LCS's. We have

(1) $\mathcal{O}^{b}(\mathcal{T}(\mathfrak{A})) \subseteq \mathfrak{A}^{\text {inj }} \subseteq \mathcal{O}(\mathcal{T}(\mathfrak{A}))$

(2) $\mathcal{O}^{b}(\mathcal{B}(\mathfrak{A})) \subseteq \mathfrak{A}^{\text {bsur }} \subseteq \mathcal{O}(\mathcal{B}(\mathfrak{A}))$. 
Proof. Let $T$ be a (topologically) bounded linear operator from a LCS $X$ into a LCS $Y$, i.e., $T \in \mathfrak{L}^{b}(X, Y)$. Then there is a continuous seminorm $p$ of $X$ and an absolutely convex bounded subset $B$ of $Y$ such that $T$ sends $V_{p}=\{x \in X: p(x) \leq 1\}$ into $B$. It is plain that $T$ has a decomposition

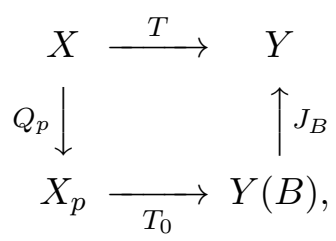

where $T_{0} \in \mathfrak{L}\left(X_{p}, Y(B)\right)$ is the unique bounded operator induced by $T$.

If $T \in \mathcal{O}^{b}(\mathfrak{T}(\mathfrak{A}))(X, Y)$ then $p$ can be chosen to be an $\mathfrak{A}$-seminorm of $X$. By Theorem 4.5. $Q_{p} \in \mathfrak{A}^{\operatorname{inj}}\left(X, X_{p}\right)$ and hence $T=J_{B} T_{0} Q_{p} \in \mathfrak{A}^{\operatorname{inj}}(X, Y)$. Similarly, if $\left.T \in \mathcal{O}^{b}(\mathcal{M})\right)(X, Y)$ then $B$ can be chosen to be an $\mathfrak{A}$-bounded subset of $Y$. By Theorem 4.5 again, $J_{B} \in \mathfrak{A}^{\text {bsur }}(Y(B), Y)$ and hence $T=J_{B} T_{0} Q_{p} \in \mathfrak{A}^{\text {bsur }}(X, Y)$. In other words, $\mathcal{O}^{b}(\mathfrak{T}(\mathfrak{A})) \subseteq \mathfrak{A}^{\text {inj }}$ and $\mathcal{O}^{b}(\mathcal{B}(\mathfrak{A})) \subseteq \mathfrak{A}^{\text {bsur }}$. The other inclusions follows from the injectivity of $\mathcal{O}(\mathcal{T}(\mathfrak{A}))$ and the bornological surjectivity of $\mathcal{O}(\mathcal{T}(\mathfrak{A}))$.

Proposition 4.8. Let $\mathcal{P}$ be a generating topology on LCS's. If the operator ideal $\mathfrak{A}=\mathcal{O}(\mathcal{P})$ is symmetric (resp. $\mathfrak{A}=\mathcal{O}^{b}(\mathcal{P})$ is symmetric) then

$$
\mathcal{P}^{\circ}(Y)=\mathcal{B}(\mathcal{O}(\mathcal{P}))(Y) \quad\left(\operatorname{resp} . \mathcal{P}^{\circ}(Y)=\mathcal{B}\left(\mathcal{O}^{b}(\mathcal{P})\right)(Y)\right)
$$

for all infrabarrelled LCS $Y$.

Proof. Let $B$ be a bounded disk in $Y$. Suppose firstly that $B$ is $\mathfrak{A}$-bounded. Then there is a normed space $N$ such that $T U_{N} \supseteq B$. Hence $B^{\circ} \supseteq\left(T^{\prime}\right)^{-1} U_{N^{\prime}}$. Now, the symmetry of $\mathfrak{A}$ implies $T^{\prime} \in \mathfrak{A}\left(Y_{\beta}^{\prime}, N^{\prime}\right)$. Thus, $B^{\circ}$ is an $\mathfrak{A}$-neighborhood of zero of $Y_{\beta}^{\prime}$. It follows from $\mathcal{T}(\mathfrak{A})=\mathcal{T}(\mathcal{O}(\mathcal{P}))=\mathcal{T}\left(\mathcal{O}^{b}(\mathcal{P})\right)=\mathcal{P}$ that $B^{\circ}$ is $\mathcal{P}\left(Y_{\beta}^{\prime}\right)$-neighborhood of zero of $Y_{\beta}^{\prime}$. Hence $B$ is $\mathcal{P}^{\circ}(Y)$-bounded.

Conversely, assume that $B$ is $\mathcal{P}^{\circ}$-bounded in $Y$. In other words, $B^{\circ}$ is an $\mathfrak{A}$-neighborhood of zero of $Y_{\beta}^{\prime}$. Therefore, there is a Banach space $F$ and a $T$ in $\mathfrak{A}\left(Y_{\beta}^{\prime}, F\right)$ such that $B^{\circ} \supseteq T^{-1} U_{F}$. Hence the second polar $B^{\circ \bullet}$ of $B$ in $Y_{\beta \beta}^{\prime \prime}$ is $\mathfrak{A}$-bounded since $B^{\circ \bullet} \in T^{\prime} U_{F^{\prime}}$ and $T^{\prime} \in \mathfrak{A}\left(F^{\prime}, Y_{\beta \beta}^{\prime \prime}\right)$. Let $K_{Y}$ be the canonical embedding of $Y$ into $Y_{\beta \beta}^{\prime \prime}$. The infrabarrelledness of $Y$ ensures that $K_{Y}$ is a topological injection. As a result, the inclusion $K_{Y} B \subseteq B^{\circ}$ establishes the existence of a $k_{B}$ in $\mathfrak{L}\left(Y(B), Y_{\beta \beta}^{\prime \prime}\left(B^{\circ \bullet}\right)\right)$ such that $J_{B^{\circ} \bullet k_{B}}=K_{X} J_{B}$. Then $J_{B} \in\left(\mathfrak{A}^{\text {bsur }}\right)^{\operatorname{inj}}(Y(B), Y)$ because $J_{B^{\circ} \bullet} \in \mathfrak{A}^{\text {bsur }}\left(Y_{\beta \beta}^{\prime \prime}\left(B^{\circ \bullet}\right), Y_{\beta \beta}^{\prime \prime}\right)$ by Theorem 4.5. However, $\left(\mathfrak{A}^{\text {bsur }}\right)^{\text {inj }}=\left(\mathfrak{A}^{\text {inj }}\right)^{\text {bsur }}=\mathfrak{A}^{\text {bsur }}$ since $\mathfrak{A}=\mathcal{O}(\mathcal{P})$ (or $\left.\mathfrak{A}=\mathcal{O}^{b}(\mathcal{P})\right)$ is always injective. This implies that $B$ is $\mathfrak{A}$-bounded, i.e., $\mathcal{B}(\mathcal{O}(\mathcal{P})$ )-bounded in $Y$, by Theorem 4.5 again. 
Proposition 4.9. Let $\mathcal{M}$ be a generating bornology on LCS's. If the operator ideal $\mathfrak{A}=\mathcal{O}(\mathcal{M})$ is symmetric (resp. $\mathfrak{A}=\mathcal{O}^{b}(\mathcal{M})$ is symmetric) then

$$
\mathcal{M}^{\circ}(X)=\mathcal{T}(\mathcal{O}(\mathcal{M}))(X) \quad\left(\operatorname{resp} . \mathcal{M}^{\circ}(X)=\mathcal{T}\left(\mathcal{O}^{b}(\mathcal{M})\right)(X)\right)
$$

for all infrabarrelled LCS X.

Proof. Let $V$ be a closed, absolutely convex neighborhood of zero of $X$. Suppose firstly that $V$ is an $\mathfrak{A}$-neighborhood of $X$ then there is a normed space $N$ and an $T$ in $\mathfrak{A}(X, N)$ such that $T^{-1} U_{N} \subseteq V$. Hence $V^{\circ} \subseteq T^{\prime} U_{N^{\prime}}$ and thus $V^{\circ}$ is $\mathcal{M}$-bounded in $X_{\beta}^{\prime}$ since $T^{\prime} \in \mathfrak{A}\left(N^{\prime}, X_{\beta}^{\prime}\right)$. So $V$ is an $\mathcal{M}^{\circ}(X)$-bounded subset of $X$.

Conversely, assume that $V$ is an $\mathcal{M}^{\circ}$-neighborhood of zero of $X$. Then $V^{\circ}$ is $\mathcal{M}\left(X_{\beta}^{\prime}\right)$-bounded in the strong dual space $X_{\beta}^{\prime}$ of $X$. Hence there is a normed space $N$ and an $T$ in $\mathfrak{A}\left(N, X_{\beta}^{\prime}\right)$ such that $T U_{N} \supseteq V^{\circ}$. Consequently, $V^{\circ} \subseteq\left(T^{\prime}\right)^{-1} U_{N^{\prime}}$ and thus $V^{\circ}$ is an $\mathfrak{A}$-neighborhood of zero of $X_{\beta \beta}^{\prime \prime}$ as $T^{\prime} \in \mathfrak{A}\left(X_{\beta \beta}^{\prime \prime}, N^{\prime}\right)$. Since $X$ is infrabarrelled, $K_{X}$ is continuous. By $\left(\mathrm{GT}_{2}\right)$, $V=K_{X}^{-1} V^{\circ \bullet}=V^{\circ \bullet} \cap X$ is an $\mathfrak{A}$-neighborhood of zero of $X$.

Definition 4.10. A generating topology $\mathcal{P}$ on $L C S$ 's is said to have the subspace property if whenever $Y$ is a subspace of a $L C S X, Y_{\mathcal{P}}$ is also a subspace of $X_{\mathcal{P}}$, i.e., the $\mathcal{P}$-topology of $Y$ coincides with the subspace topology inherited from the $\mathcal{P}$-topology of $X$. See Jarchow 8 for the Banach space version.

Let $\mathfrak{A}$ be an operator ideal on LCS's or Banach spaces. $\mathfrak{A}^{\text {dual }}$ denotes the operator ideal with components

$$
\mathfrak{A}^{\text {dual }}(X, Y)=\left\{T \in \mathcal{L}(X, Y): T^{\prime} \in \mathfrak{A}\left(Y_{\beta}^{\prime}, X_{\beta}^{\prime}\right)\right\}
$$

Proposition 4.11. Let $\mathcal{P}$ be a generating topology on LCS's and $X$ be an infrabarrelled LCS. Then

(a) $\mathcal{O}^{b}(\mathcal{P})^{\text {dual }}(X, Y)=\mathcal{O}^{b}\left(\mathcal{P}^{\circ}\right)(X, Y), \quad \forall L C S Y$.

(b) $\mathcal{O}^{b}\left(\mathcal{P}^{\circ}\right)^{\mathrm{dual}}(X, Y) \subseteq \mathcal{O}^{b}(\mathcal{P})(X, Y), \quad \forall L C S Y$.

If, in addition, $\mathcal{O}(\mathcal{P})$ is symmetric or $\mathcal{P}$ has the subspace property then

$(b)^{\prime} \mathcal{O}^{b}\left(\mathcal{P}^{\circ}\right)^{\operatorname{dual}}(X, Y)=\mathcal{O}^{b}(\mathcal{P})(X, Y), \quad \forall L C S Y$.

Proof. (a) Let $T \in \mathcal{O}^{b}(\mathcal{P})^{\text {dual }}(X, Y)$, i.e., $T^{\prime} \in \mathcal{O}^{b}(\mathcal{P})\left(Y_{\beta}^{\prime}, X_{\beta}^{\prime}\right)$. Then there is a $\mathcal{P}\left(Y_{\beta}^{\prime}\right)$-neighborhood $V$ of 0 in $Y_{\beta}^{\prime}$ such that $T^{\prime} V$ is bounded in $X_{\beta}^{\prime}$. Hence $U=\left(T^{\prime} V\right)^{\circ}=T^{-1} V^{\circ}$ is a closed bornivorous barrel in $X$. Since $X$ is infrabarrelled, $U$ is a 0 -neighborhood in $X$. Now 
$T U \subseteq V^{\circ}$ ensures that $T \in \mathcal{O}^{b}\left(\mathcal{P}^{\circ}\right)(X, Y)$. Conversely, if $T \in \mathcal{O}^{b}\left(\mathcal{P}^{\circ}\right)(X, Y)$ then there is a 0 -neighborhood $U$ in $X$ such that $A=T U$ is $\mathcal{P}^{\circ}(Y)$-bounded in $Y$. Hence $A^{\circ}=\left(T^{\prime}\right)^{-1} U^{\circ}$ is a $\mathcal{P}\left(Y_{\beta}^{\prime}\right)$-neighborhood of 0 in $Y_{\beta}^{\prime}$. Now $T^{\prime} A^{\circ} \subseteq U^{\circ}$ implies that $T^{\prime} \in \mathcal{O}^{b}(\mathcal{P})\left(Y_{\beta}^{\prime}, X_{\beta}^{\prime}\right)$.

(b) Let $T \in \mathcal{O}^{b}\left(\mathcal{P}^{\circ}\right)^{\text {dual }}(X, Y)$, i.e., $T^{\prime} \in \mathcal{O}^{b}\left(\mathcal{P}^{\circ}\right)\left(Y_{\beta}^{\prime}, X_{\beta}^{\prime}\right)$. Then there is a 0 -neighborhood $V$ in $Y_{\beta}^{\prime}$ such that $T^{\prime} V$ is $\mathcal{P}^{\circ}\left(X_{\beta}^{\prime}\right)$-bounded in $X_{\beta}^{\prime}$. Hence $U=\left(T^{\prime} V\right)^{\bullet}$ is a $\mathcal{P}\left(X_{\beta \beta}^{\prime \prime}\right)$-neighborhood of 0 in $X_{\beta \beta}^{\prime \prime}$. Let $U_{0}=K_{X}^{-1} U$. By the functorial property $\left(G T_{2}\right)$ of $\mathcal{P}, U_{0}$ is a $\mathcal{P}(X)-$ neighborhood of 0 in $X$. It is easy to see that $T U_{0} \subseteq V^{\circ}$ and thus $T \in \mathcal{O}^{b}(\mathcal{P})(X, Y)$.

(b)' Assume, in addition to those in (b), that $\mathfrak{A}=\mathcal{O}(\mathcal{P})$ is symmetric. Let $T \in \mathcal{O}^{b}(\mathcal{P})(X, Y)$. We want to verify that $T^{\prime} \in \mathcal{O}^{b}\left(\mathcal{P}^{\circ}\right)\left(Y_{\beta}^{\prime}, X_{\beta}^{\prime}\right)$. By assumption, there is a $\mathcal{P}(X)$-neighborhood $U$ of 0 in $X$ such that $A=T U$ is bounded in $Y$. Now $A^{\circ}=\left(T^{\prime}\right)^{-1} U^{\circ}$ suggests us to check if $U^{\circ}$ is $\mathcal{P}^{\circ}\left(X_{\beta}^{\prime}\right)$-bounded in $X_{\beta}^{\prime}$. Since $\mathcal{P}=\mathcal{T}(\mathcal{O}(\mathcal{P}))=\mathcal{T}(\mathfrak{A})$, there is a Banach space $F$ and an $R$ in $\mathfrak{A}(X, F)$ such that $U \supseteq R^{-1} U_{F}$. Therefore, $U^{\circ} \subseteq R^{\prime} U_{F^{\prime}}$ and thus $U^{\circ} \supseteq\left(\left(R^{\prime}\right)^{\prime}\right)^{-1} U_{F^{\prime \prime}}$ where $\left(R^{\prime}\right)^{\prime}$ is the double adjoint of $R$ from $X_{\beta \beta}^{\prime \prime}$ into $F^{\prime \prime}$. Since $\mathfrak{A}$ is symmetric, $\left(R^{\prime}\right)^{\prime} \in \mathfrak{A}\left(X_{\beta \beta}^{\prime \prime}, F^{\prime \prime}\right)$

and thus $U^{\circ}$ is a $\mathcal{P}\left(X_{\beta \beta}^{\prime \prime}\right)$-neighborhood of 0 in $X_{\beta \beta}^{\prime \prime}$. Consequently, $U^{\circ}$ is a $\mathcal{P}^{\circ}\left(X_{\beta}^{\prime}\right)$-bounded subset of $X_{\beta}^{\prime}$, as asserted.

Finally, if the subspace property of $\mathcal{P}$ is assumed instead of the symmetry of $\mathcal{O}(\mathcal{P})$ then the $\mathcal{P}(X)$-neighborhood $U$ of 0 in $X$ above is induced from a $\mathcal{P}\left(X_{\beta \beta}^{\prime \prime}\right)$-neighborhood $V$ of 0 in $X_{\beta \beta}^{\prime \prime}$, i.e., $K_{X} U=V \cap K_{X} X$ and thus $U^{\circ}=V^{\bullet}$ is $\mathcal{P}^{\circ}\left(X_{\beta}^{\prime}\right)$-bounded in $X_{\beta}^{\prime}$, where $K_{X}$ is the evaluation map from $X$ into $X_{\beta \beta}^{\prime \prime}$.

\section{LCS'S DEFINED BY OPERATORS, TOPOLOGIES AND BORNOLOGIES}

Theorem 5.1. Let $\mathfrak{A}$ be an operator ideal on LCS's and $X$ be a LCS. The following are all equivalent.

(1) $X$ is $\mathfrak{A}$-topological.

(2) For each continuous seminorm $p$ on $X, Q_{p} \in \mathfrak{A}^{\mathrm{inj}}\left(X, X_{p}\right)$, or equivalently, $\widetilde{Q}_{p} \in \mathfrak{A}^{\mathrm{inj}}\left(X, \widetilde{X}_{p}\right)$.

(3) $\mathcal{L}^{b}(X, Y) \subseteq \mathfrak{A}^{\mathrm{inj}}(X, Y)$ for every LCS $Y$.

(4) $\mathcal{L}(X, F)=\mathfrak{A}^{\operatorname{inj}}(X, F)$ for every normed (or Banach) space $F$.

(5) $i d_{X} \in \mathcal{L}\left(X_{\mathfrak{A}}, X\right)$, where $X_{\mathfrak{A}}$ is the LCS X equipped with the $\mathfrak{A}$-topology.

Proof. (1) $\Leftrightarrow(2)$ is contained in Theorem 4.5] (1) $\Leftrightarrow(5)$ and $(2) \Rightarrow(3) \Rightarrow(4)$ are trivial. (4) $\Rightarrow(1)$ is due to Theorem 4.5 again. 
In the following, $\mathcal{C}$ denotes either the class of all LCS's or the class of all Banach spaces. The next result is a generalization of a result of Jarchow [9, Proposition 3].

Theorem 5.2 (26]). Let $\mathfrak{A}$ be a surjective operator ideal on $\mathcal{C}$. If $X, Y \in \mathcal{C}$ and $Y$ is a (topological) quotient space of $X$ then the $\mathfrak{A}$-topology of $Y$ is the quotient topology induced by the $\mathfrak{A}$-topology of $X$. In particular, a quotient space of an $\mathfrak{A}$-topological space is again an $\mathfrak{A}$-topological space.

Theorem 5.3. Let $\mathfrak{A}$ be an operator ideal on $L C S$ 's and $Y$ be a LCS. The following are all equivalent.

(1) $Y$ is $\mathfrak{A}$-bornological.

(2) $J_{B} \in \mathfrak{A}^{\mathrm{bsur}}(Y(B), Y)$ for each bounded disk $B$ in $Y$.

(3) $\mathcal{L}^{b}(X, Y) \subseteq \mathfrak{A}^{\text {bsur }}(X, Y)$ for every $L C S X$.

(4) $\mathcal{L}(N, Y)=\mathfrak{A}^{\mathrm{bsur}}(N, Y)$ for every normed space $N$.

(5) $i d_{Y} \in L^{\times}\left(Y, Y^{\mathfrak{A}}\right)$, where $Y^{\mathfrak{A}}$ is the convex bornological vector space $Y$ equipped with the $\mathfrak{A}$-bornology.

In case $Y$ is infracomplete they are all equivalent to

$(4)^{\prime} \mathcal{L}(E, Y)=\mathfrak{A}^{\mathrm{bsur}}(E, Y)$ for every Banach space $E$.

If $\mathfrak{A}$ is surjective we can replace $\mathfrak{A}^{\text {bsur }}$ by $\mathfrak{A}$ in all of the above statements.

Proof. (1) $\Leftrightarrow(5)$ is by definition. It is plain that $(2) \Rightarrow(3) \Rightarrow(4) \Rightarrow(4)^{\prime}$. (4) $\Rightarrow(1)$ (or $(4)^{\prime} \Rightarrow 1$ in case $Y$ is infracomplete) and $(1) \Leftrightarrow(2)$ is due to Theorem 4.5. The last assertion is a consequence of Proposition 3.2

Let $\mathcal{C}$ be either the class of all LCS's or the class of all Banach spaces.

Theorem 5.4. 26] Let $\mathfrak{A}$ be an injective operator ideal on $\mathcal{C}$ and $X, Y \in \mathcal{C}$. If $Y$ is a (topological) subspace of $X$ then the $\mathfrak{A}$-bornology of $Y$ is the subspace bornology inherited from the $\mathfrak{A}$-bornology of $X$. In particular, a subspace of an $\mathfrak{A}$-bornological space is again an $\mathfrak{A}-$ bornological space.

Theorem 5.5. Let $\mathfrak{A}$ be an operator ideal on LCS's. Let $\mathcal{P}=\mathcal{T}(\mathfrak{A})$ be the ideal topology on $L C S$ 's generated by $\mathfrak{A}$. A LCS X is $\mathfrak{A}$-topological if and only if

$$
\mathcal{L}^{b}(X, Y) \cap \mathcal{O}(\mathcal{P})(X, Y)=\mathcal{O}^{b}(\mathcal{P})(X, Y)
$$

for each $L C S Y$. 
Proof. By Theorem 5.1] if $X$ is $\mathfrak{A}$-topological then $\mathcal{O}^{b}(\mathcal{P})(X, Y)=\mathcal{L}^{b}(X, Y)$ and $\mathcal{O}(\mathcal{P})(X, Y)=$ $\mathcal{L}(X, Y)$. The equality follows. Conversely, assume the equality holds for every LCS $Y$. It suffices to show that $\mathcal{L}(X, N) \subseteq \mathcal{O}^{b}(\mathcal{P})(X, N)$ for each normed space. Let $N_{\mathcal{P}}$ be the LCS given by equipping $N$ with the $\mathcal{P}(N)$-topology. By the functorial property $\left(G T_{2}\right)$ of $\mathcal{P}$, any $T$ in $\mathcal{L}(X, N)$ also belongs to $\mathcal{L}\left(X_{\mathcal{P}}, N_{\mathcal{P}}\right)=\mathcal{O}(\mathcal{P})\left(X, N_{\mathcal{P}}\right)$. By the hypothesis, $T \in \mathcal{O}^{b}(\mathcal{P})\left(X, N_{\mathcal{P}}\right)$. Since $\mathcal{P}(N)$ is compatible with the dual pair $\left(N, N^{\prime}\right)$ by $\left(G T_{1}\right)$, we have $T \in \mathcal{O}^{b}(\mathcal{P})(X, N)$. It follows the desired assertion.

Remark 5.6. If we let $\mathcal{M}=\mathcal{B}(\mathfrak{A})$ then a LCS $Y$ being $\mathfrak{A}$-bornological implies

$$
\mathcal{L}^{b}(X, Y) \cap \mathcal{O}(\mathcal{M})(X, Y)=\mathcal{O}^{b}(\mathcal{M})(X, Y)
$$

for each LCS $X$. We do not know if the converse is true.

Let $\mathfrak{A}$ be an operator ideal on LCS's. Denote by $\mathfrak{A}_{\mathbb{B}}$ the operator ideal defined on Banach spaces such that $\mathfrak{A}_{\mathbb{B}}(E, F)=\mathfrak{A}(E, F)$ for every pair $E$ and $F$ of Banach spaces. Conversely, let $\mathfrak{A}$ be an operator ideal on Banach spaces. There are many ways to extend $\mathfrak{A}$ to an operator ideal $\mathfrak{A}_{0}$ on LCS's in the sense that $\left(\mathfrak{A}_{0}\right)_{\mathbb{B}}=\mathfrak{A}$. In [15], Pietsch mentioned six different ways to extend $\mathfrak{A}$ to an operator ideal on LCS's. Among them, we are interested in

$$
\begin{aligned}
\mathfrak{A}^{\text {inf }} & =\left\{R S_{0} T \in \mathfrak{L}(X, Y): T \in \mathfrak{L}\left(X, X_{0}\right), S_{0} \in \mathfrak{A}\left(X_{0}, Y_{0}\right), R \in \mathfrak{L}\left(Y_{0}, Y\right)\right\}, \\
\mathfrak{A}^{\text {rup }} & =\left\{S \in \mathfrak{L}(X, Y): \forall B \in \mathfrak{L}\left(Y, Y_{0}\right), \exists A \in \mathfrak{L}\left(X, X_{0}\right), S_{0} \in \mathfrak{A}\left(X_{0}, Y_{0}\right) \text { such that } B S=S_{0} A\right\}, \\
\mathfrak{A}^{\text {lup }}= & \left\{S \in \mathfrak{L}(X, Y): \forall B \in \mathfrak{L}\left(X_{0}, X\right), \exists A \in \mathfrak{L}\left(Y_{0}, Y\right), S_{0} \in \mathfrak{A}\left(X_{0}, Y_{0}\right) \text { such that } S B=A S_{0}\right\}, \\
\mathfrak{A}^{\text {sup }}= & \left\{S \in \mathfrak{L}(X, Y): R S T \in \mathfrak{A}\left(X_{0}, Y_{0}\right), \text { for all } T \in \mathfrak{L}\left(X_{0}, X\right) \text { and } R \in \mathfrak{L}\left(Y, Y_{0}\right)\right\} .
\end{aligned}
$$

Here, $X, Y$ run through all LCS's and $X_{0}, Y_{0}$ run through all Banach spaces.

Definition $5.7([26])$. Let $\mathfrak{A}$ be an operator ideal on Banach spaces. We call a continuous seminorm $p$ on a LCS $X$ a $\operatorname{Groth}(\mathfrak{A})$-seminorm if there is a continuous seminorm $q$ on $X \operatorname{such}$ that $p \leq q$ and $\widetilde{Q}_{p q} \in \mathfrak{A}\left(\widetilde{X}_{q}, \widetilde{X}_{p}\right)$. The $\operatorname{Groth}(\mathfrak{A})$-topology on $X$ is defined to be the locally convex (Hausdorff) topology on $X$ which has a subbase determined by all $\operatorname{Groth}(\mathfrak{A})$-seminorms.

A LCS $X$ is a $\operatorname{Groth}(\mathfrak{A})$-space if its topology coincides with the $\operatorname{Groth}(\mathfrak{A})$-topology. It is equivalent to say that the identity map $i d_{X} \in \mathfrak{A}^{\text {rup }}(X, X)$.

Let $\mathcal{P}$ be a generating topology on Banach spaces. Define $\mathcal{P}^{\mathbb{L}}(X)$ on each LCS $X$ to be the coarsest locally convex (Hausdorff) topology on $X$ among those $\mathcal{P}_{0}(X)$ such that the inclusion

$$
\mathcal{L}(X, F) \subseteq \mathcal{L}\left(X_{\mathcal{P}_{0}}, F_{\mathcal{P}}\right)
$$


holds for every Banach space $F$. It is clear that for each LCS $X, \mathcal{P}_{\sigma}(X) \leq \mathcal{P}^{\mathbb{L}}(X) \leq \mathcal{P}_{\text {ori }}(X)$ and a continuous seminorm $p$ on $X$ is $\mathcal{P}^{\mathbb{L}}(X)$-continuous if and only if there is a Banach space $F$, an $S$ in $\mathcal{L}(X, F)$ and a $\mathcal{P}(F)$-continuous seminorm $r$ on $F$ such that $p(x) \leq r(S x)$ for all $x$ in $X$.

Lemma 5.8. $\mathcal{P}^{\mathbb{L}}=\left\{\mathcal{P}^{\mathbb{L}}(X): X L C S\right\}$ is the minimal extension of $\mathcal{P}$ to LCS's.

Proof. It is easy to see that $\mathcal{P}^{\mathbb{L}}$ is a generating topology on LCS's. Let $E$ be a Banach space. By definition of $\mathcal{P}^{\mathbb{L}}, \mathcal{P}^{\mathbb{L}}(E) \leq \mathcal{P}(E)$. On the other hand, $i d_{E} \in \mathcal{L}(E, E) \subseteq \mathcal{L}\left(E_{\mathcal{P} \mathbb{L}}, E_{\mathcal{P}}\right)$ implies $\mathcal{P}^{\mathbb{L}}(E) \geq \mathcal{P}(E)$. So $\mathcal{P}^{\mathbb{L}}$ is an extension of $\mathcal{P}$ to LCS's. The minimality of $\mathcal{P}^{\mathbb{L}}$ is obvious.

Theorem 5.9. Let $\mathfrak{A}$ be an operator ideal on Banach spaces. The minimal extension $\mathcal{P}^{\mathbb{L}}$ of $\mathcal{P}=\mathcal{T}(\mathfrak{A})$ coincides with the $\operatorname{Groth}(\mathfrak{A})$-topology.

Proof. Without loss of generality, we can assume that $\mathfrak{A}$ is injective since $\mathcal{T}(\mathfrak{A})=\mathcal{T}\left(\mathfrak{A}^{\mathrm{inj}}\right)$ by Theorem 4.5. Let $p=r \circ S$ be a $\mathcal{P}^{\mathbb{L}}$-continuous seminorm on a LCS $X$ where $S \in \mathcal{L}(X, F)$ and $r$ is a $\mathcal{P}$-continuous seminorm on a Banach space $F$. Then we have $\left\|\widetilde{Q}_{p}(x)\right\|_{\widetilde{X}_{p}}=\left\|\widetilde{Q}_{r}(S x)\right\|_{\widetilde{F}_{r}}$ for all $x$ in $X$. It follows that there is an isometry $S_{0}$ in $\mathcal{L}\left(\widetilde{X}_{p}, \widetilde{F}_{r}\right)$ such that $S_{0} \widetilde{Q}_{p}=\widetilde{Q}_{r} S$. Note that $\widetilde{Q}_{r} \in \mathfrak{A}\left(F, \widetilde{F}_{r}\right)$. Define a continuous seminorm $q$ on $X$ by $q(x)=\left\|\widetilde{Q}_{r}\right\|\|S x\|$. Now $q(x) \geq\left\|\widetilde{Q}_{r} S x\right\|=p(x)$ and we have an $S_{2}$ in $\mathcal{L}\left(\widetilde{X}_{q}, F\right)$ induced by $S$. Since $S_{0}$ is an injection and $S_{0} \widetilde{Q}_{p q}=\widetilde{Q}_{r} S_{2} \in \mathfrak{A}\left(\widetilde{X}_{q}, \widetilde{F}_{r}\right), \widetilde{Q}_{p q} \in \mathfrak{A}\left(\widetilde{X}_{q}, \widetilde{X}_{p}\right)$, i.e., $p$ is a $\operatorname{Groth}(\mathfrak{A})$-seminorm.

Conversely, if $p$ is a $\operatorname{Groth}(\mathfrak{A})$-continuous seminorm on $X$ then there is a continuous seminorm $q$ on $X$ with $p \leq q$ such that $\widetilde{Q}_{p q} \in \mathfrak{A}\left(\widetilde{X}_{q}, \widetilde{X}_{p}\right)=\mathcal{L}\left(\left(\widetilde{X}_{q}\right)_{\mathcal{P}}, \widetilde{X}_{p}\right)$ by Theorem 2.2(4a). In other words, the seminorm $r$ on $\widetilde{X}_{q}$ defined by $r(y)=\left\|\widetilde{Q}_{p q}(y)\right\|_{\widetilde{X}_{p}}, y \in \widetilde{X}_{q}$, is $\mathcal{P}$-continuous. Note that $\widetilde{Q}_{p}=\widetilde{Q}_{p q} \widetilde{Q}_{q}$ implies that $p(x)=\left\|\widetilde{Q}_{p q} \widetilde{Q}_{q}(x)\right\|=r\left(\widetilde{Q}_{q} x\right)$. It simply says that $p$ is a $\mathcal{P}^{\mathbb{L}}$-continuous seminorm.

Theorem 5.10. Let $\mathfrak{A}$ be an operator ideal on Banach spaces with $\mathcal{P}=\mathcal{T}(\mathfrak{A})$. Then $\mathcal{O}\left(\mathcal{P}^{\mathbb{L}}\right)=$ $\left(\mathfrak{A}^{\text {inj }}\right)^{\text {rup }}$.

Proof. Let $X$ and $Y$ be LCS's. Assume $T \in \mathcal{O}\left(\mathcal{P}^{\mathbb{L}}\right)(X, Y)$. Then for every Banach space $F$ and $S$ in $\mathcal{L}(Y, F), S T \in \mathcal{O}\left(\mathcal{P}^{\mathbb{L}}\right)(X, F)=\mathcal{L}\left(X_{\mathcal{P} \mathbb{L}}, F\right)$. Hence there is a $\mathcal{P}^{\mathbb{L}}$-continuous seminorm $p$ on $X$ such that $\|S T x\| \leq p(x)$. By Theorem[5.9, there is a continuous seminorm $q$ on $X$ such that $p \leq q$ and $\widetilde{Q}_{p q} \in \mathfrak{A}^{\operatorname{inj}}\left(\widetilde{X}_{q}, \widetilde{X}_{p}\right)$. Let $R \in \mathfrak{L}\left(\widetilde{X}_{p}, F\right)$ is induced by the inequality $\|S T x\| \leq p(x)$. It is then not difficult to see that $S T=R \widetilde{Q}_{p q} \widetilde{Q}_{q}$, and thus $T \in\left(\mathfrak{A}^{\mathrm{inj}}\right)^{\operatorname{rup}}(X, Y)$. 
Conversely, assume $T \in\left(\mathfrak{A}^{\mathrm{inj}}\right)^{\mathrm{rup}}(X, Y)$. Then for every continuous seminorm $p$ on $Y$ there exists a Banach space $E$, an $R$ in $\mathcal{L}(X, E)$ and an $S$ in $\mathfrak{A}^{\operatorname{inj}}\left(E, \widetilde{Y}_{p}\right)$ such that $\widetilde{Q}_{p} T=S R$. Now $S \in \mathcal{L}\left(E_{\mathcal{P}}, \widetilde{Y}_{p}\right)$ and $R \in \mathcal{L}(X, E) \subseteq \mathcal{L}\left(X_{\mathcal{P L}}, E_{\mathcal{P}}\right)$ imply $\widetilde{Q}_{p} T=S R \in \mathcal{L}\left(X_{\mathcal{P} \mathbb{L}}, \widetilde{Y}_{p}\right)$. Since it is true for every continuous seminorm $p$ on $Y, T \in \mathcal{L}\left(X_{\mathcal{P} \mathbb{L}}, Y\right)$, i.e., $T \in \mathcal{O}\left(\mathcal{P}^{\mathbb{L}}\right)(X, Y)$.

Definition 5.11 ([26]). Let $\mathfrak{A}$ be an operator ideal on Banach spaces. A bounded $\sigma$-disk $A$ in a LCS $X$ is said to be $\operatorname{Groth}(\mathfrak{A})$-bounded in $X$ if there is a bounded $\sigma$-disk $B$ in $X$ such that $A \subseteq B$ and the canonical map $J_{B A} \in \mathfrak{A}(X(A), X(B))$. Note that, in this case, both $X(A)$ and $X(B)$ are Banach spaces. The Groth( $(\mathfrak{A})$-bornology on a LCS $X$ is defined to be the convex vector bornology on $X$ with a subbase consisting of $\operatorname{Groth}(\mathfrak{A})$-bounded $\sigma$-disks in $X$.

A LCS is a co-Groth( $(\mathfrak{A})$-space, if all bounded $\sigma$-disks in $X$ are $\operatorname{Groth}(\mathfrak{A})$-bounded. It is equivalent to say that $i d_{X} \in \mathfrak{A}^{\operatorname{lup}}(X, X)$.

Let $\mathcal{M}$ be a generating bornology on Banach spaces. We define, for each LCS $X$, a convex vector bornology $\mathcal{M}^{\mathbb{L}}(X)$ on $X$ to be the smallest convex (separated) vector bornology among those $\mathcal{M}_{0}$ on $X$ such that

$$
\mathcal{L}(E, X) \subseteq L^{\times}\left(E^{\mathcal{M}}, X^{\mathcal{M} \mathfrak{M}_{0}}\right)
$$

holds for every Banach space $E$. It is easy to see that $\mathcal{M}_{\text {fin }}(X) \subseteq \mathcal{M}^{\mathbb{L}}(X) \subseteq \mathcal{M}_{\text {von }}(X)$ and the family of subsets $B$ in $X$ in the form of $B=T A$ for some $T \in \mathcal{L}(E, X)$ and $\mathcal{M}$-bounded set $A$ in a Banach space $E$ forms a basis of the bornology $\mathcal{M}^{\mathbb{L}}(X)$ for each LCS $X$.

Lemma 5.12. Let $\mathcal{M}$ be a generating bornology on Banach spaces. $\mathcal{M}^{\mathbb{L}}$ is the minimal extension of $\mathcal{M}$ to $L C S$ 's.

Proof. Similar to Lemma 5.8 .

Theorem 5.13. Let $\mathfrak{A}$ be an operator ideal on Banach spaces. The minimal extension $\mathcal{M}^{\mathbb{L}}$ of $\mathcal{M}=\mathcal{B}(\mathfrak{A})$ coincides with the $\operatorname{Groth}(\mathfrak{A})$-bornology.

Proof. Without loss of generality, we can assume that $\mathfrak{A}$ is surjective since $\mathcal{B}(\mathfrak{A})=\mathcal{B}\left(\mathfrak{A}^{\text {sur }}\right)$ by Theorem 4.5. Let $A$ be a $\operatorname{Groth}(\mathfrak{A})$-bounded $\sigma$-disk in a LCS $X$. By definition, there is a bounded $\sigma$-disk $B$ in $X$ such that $A \subseteq B$ and $J_{B A} \in \mathfrak{A}(X(A), X(B))=L^{\times}\left(X(A), X(B)^{\mathcal{M}}\right)$. In other words, $C=J_{B A} U_{X(A)}$ is $\mathcal{M}$-bounded in $X(B)$. Now $A \subseteq J_{A} U_{X(A)}=J_{B} J_{B A} U_{X(A)}=$ $J_{B} C$ implies that $A$ is $M^{\mathbb{L}}$-bounded.

Conversely, if $A=S B$ is $\mathcal{M}^{\mathbb{L}}$-bounded in $X$ with some $S$ in $\mathcal{L}(E, X)$ and $\mathcal{M}$-bounded $\sigma$-disk $B$ in a Banach space $E$. Let $C=\lambda S U_{E}$ for some $\lambda>0$ such that $\lambda U_{E} \supseteq B$. We have $C \supseteq A$. 
Let $S_{0} \in \mathfrak{L}(E(B), X(A))$ and $S_{2} \in \mathfrak{L}(E, X(C))$ be induced by $S$. Since $B$ is $\mathcal{M}$-bounded in $E$, $J_{B} \in \mathfrak{A}(E(B), E)$ and $J_{C A} S_{0}=S_{2} J_{B} \in \mathfrak{A}(E(B), X(C))$. Finally the surjectivity of $S_{0}$ ensures that $J_{C A} \in \mathfrak{A}(X(A), X(C))$.

Theorem 5.14. Let $\mathfrak{A}$ be an operator ideal on Banach spaces with $\mathcal{M}=\mathcal{B}(\mathfrak{A})$. Then

$$
\mathcal{O}\left(\mathcal{M}^{\mathbb{L}}\right)(X, Y) \subseteq\left(\mathfrak{A}^{\text {sur }}\right)^{\operatorname{lup}}(X, Y), \quad \forall L C S \text { 's } X, Y
$$

If $X$ is infracomplete (in particular, a Banach space) then we have

$$
\mathcal{O}\left(\mathcal{M}^{\mathbb{L}}\right)(X, Y)=\left(\mathfrak{A}^{\text {sur }}\right)^{\operatorname{lup}}(X, Y), \quad \forall L C S Y .
$$

Proof. Similar to a previous theorem except that we shall use Theorem5.13 instead of Theorem [5.9. The introduction of the infracompleteness is merely to give us a chance to utilize the extension condition.

We provide a new proof for the following result.

Theorem $5.15([26])$. Let $\mathfrak{A}$ be an operator ideal on Banach spaces. The Groth $\left(\mathfrak{A}^{\mathrm{inj}}\right)$-topology coincides with the $\mathfrak{A}^{\text {rup }}$-topology on every LCS, and the Groth( $\left.\mathfrak{A}^{\text {sur }}\right)$-bornology coincides with

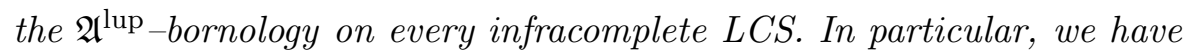

(a) A LCS X is a Groth( $\left.\mathfrak{A}^{\mathrm{inj}}\right)$-space if and only if $X$ is an $\mathfrak{A}^{\text {rup }}$-topological space.

(b) An infracomplete $L C S X$ is a co-Groth( $\left.\mathfrak{A}^{\text {sur }}\right)$-space if and only if $X$ is an $\mathfrak{A}^{\text {lup }- \text { bornological }}$ space.

(c) The $\mathfrak{A}$-topology (resp. $\mathfrak{A}$-bornology) coincides with the Groth $\left(\mathfrak{A}^{\mathrm{inj}}\right)$-topology (resp. Groth $\left(\mathfrak{A}^{\mathrm{sur}}\right)$ bornology) on Banach spaces.

Proof. Let $\mathcal{P}=\mathcal{T}(\mathfrak{A})$ and $\mathcal{M}=\mathcal{B}(\mathfrak{A})$ be the ideal topology and the ideal bornology on Banach spaces generated by $\mathfrak{A}$, respectively. Let $p$ be a continuous seminorm on a LCS $X$. We observe the following equivalences:

$p$ is a $\operatorname{Groth}\left(\mathfrak{A}^{\mathrm{inj}}\right)$-continuous seminorm on $X$.

$\Leftrightarrow p$ is an $\mathcal{O}\left(\mathcal{P}^{\mathbb{L}}\right)$-continuous seminorm on $X$ by Theorem 5.9

$\Leftrightarrow p$ is an $\left(\mathfrak{A}^{\text {inj }}\right)^{\text {rup }}$-continuous seminorm on $X$ by Theorem 5.10 ,

$\Leftrightarrow \widetilde{Q}_{p} \in\left[\left(\mathfrak{A}^{\text {inj }}\right)^{\text {rup }}\right]^{\text {inj }}\left(X, \widetilde{X}_{p}\right)$ by Theorem 4.5 .

$\Leftrightarrow \widetilde{Q}_{p} \in\left(\mathfrak{A}^{\mathrm{rup}}\right)^{\mathrm{inj}}\left(X, \widetilde{X}_{p}\right)$ by [28, Proposition 3.5].

$\Leftrightarrow p$ is an $\mathfrak{A}^{\text {rup }}$-continuous seminorm on $X$ by Theorem 4.5 . 
For the bornological case, assuming that $X$ is infracomplete, we have for each bounded $\sigma-\operatorname{disk} A$ in $X$ :

$A$ is a co-Groth $\left(\mathfrak{A}^{\text {sur }}\right)$-bounded set in $X$.

$\Leftrightarrow A$ is an $\mathcal{O}\left(\mathcal{M}^{\mathbb{L}}\right)$-bounded set in $X$ by Theorem 5.13 .

$\Leftrightarrow A$ is an $\left(\mathfrak{A}^{\text {sur }}\right)^{\text {lup }}$-bounded set in $X$ by Theorems 4.5 and 5.14

$\Leftrightarrow J_{A} \in\left[\left(\mathfrak{A}^{\text {sur }}\right)^{\operatorname{lup}}\right]^{\text {bsur }}(X(A), X)$ by Theorem 4.5.

$\Leftrightarrow J_{A} \in\left(\mathfrak{A}^{\text {lup }}\right)^{\text {bsur }}(X(A), X)$ by [28, Proposition 3.5].

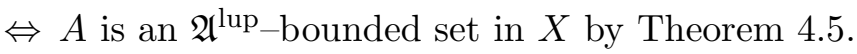

Proposition 5.16. Let $\mathcal{M}$ be a generating bornology on LCS's and $\mathfrak{A}=\mathcal{O}^{b}(\mathcal{M})$. The $\mathfrak{A}-$ topology coincides with the Grothendieck topology generated by $\mathfrak{A}$ on every LCS.

Proof. It is easy to see that $\mathfrak{A} \subseteq \mathfrak{A}_{\mathbb{B}}^{\text {rup }}$. The result follows from Theorems 5.9 and 5.15 ,

Proposition 5.17. Let $\mathcal{M}$ be a generating bornology on LCS's and $\mathfrak{A}=\mathcal{O}(\mathcal{M})$. Then the $\mathfrak{A}$-topology coincides with the $\left(\mathfrak{A}_{\mathbb{B}}^{\mathrm{inj}}\right)^{\mathrm{sup}}$-topology on each infracomplete LCS.

Proof. Since $\mathfrak{A} \subseteq\left(\mathfrak{A}_{\mathbb{B}}^{\text {inj }}\right)^{\text {sup }}, \mathcal{T}(\mathfrak{A})$ is always weaker than $\mathcal{T}\left(\left(\mathfrak{A}_{\mathbb{B}}^{\text {inj }}\right)^{\text {sup }}\right)$ on each LCS. By 28 , Corollary 3.2], $\left(\mathfrak{A}_{\mathbb{B}}^{\text {inj }}\right)^{\text {sup }}$ is injective. Let $p$ be a $\mathcal{T}\left(\left(\mathfrak{A}_{\mathbb{B}}^{\text {inj }}\right)^{\text {sup }}\right)$-continuous seminorm on an infracomplete LCS $X$. Then $\widetilde{Q}_{p} \in\left(\mathfrak{A}_{\mathbb{B}}^{\text {inj }}\right)^{\sup }\left(X, \widetilde{X}_{p}\right)$. Let $B$ be a bounded $\sigma$-disk in $X$. Now $J_{\widetilde{X}_{p}} \widetilde{Q}_{p} J_{B} \in \mathfrak{A}_{\mathbb{B}}^{\mathrm{inj}}\left(X(B), \widetilde{X}_{p}^{\mathrm{inj}}\right)$ implies $\widetilde{Q}_{p} J_{B} \in \mathfrak{A}_{\mathbb{B}}^{\mathrm{inj}}\left(X(B), \widetilde{X}_{p}\right)$ and then again implies $J_{\widetilde{X}_{p}} \widetilde{Q}_{p} J_{B} \in \mathfrak{A}_{\mathbb{B}}\left(X(B), \widetilde{X}_{p}^{\mathrm{inj}}\right)$ by Proposition 3.2 Consequently, $J_{\widetilde{X}_{p}} \widetilde{Q}_{p} \in \mathfrak{A}_{\mathbb{B}}=\mathcal{O}\left(\mathcal{M}_{\mathbb{B}}\right)$. It turns out that $\widetilde{Q}_{p} \in \mathfrak{A}^{\text {inj }}$, or equivalently, $p$ is an $\mathfrak{A}$-continuous seminorm by Theorem 4.5 .

Example 5.18. Let $X=\mathbb{K}^{(I)}$ be the locally convex direct sum of card $(I)$ many $\mathbb{K}^{\prime}$ 's where the index set $I$ is uncountable. $X$ is infracomplete. Let $\mathcal{M}_{p c}$ be the generating bornology of precompact sets (= totally bounded sets). Then $\mathcal{O}^{b}\left(\mathcal{M}_{p c}\right)=\mathfrak{K}_{p}$, the ideal of all precompact operators and $\mathcal{O}\left(\mathcal{M}_{p c}\right)=\mathfrak{K}_{p}^{\text {loc }}$, the ideal of all locally precompact operators, i.e., those sending bounded sets onto precompact sets. $\mathfrak{K}_{p}$ is surjective but not bornologically surjective and $\mathfrak{K}_{p}^{\text {loc }}$ is bornologically surjective. Now $i d_{X} \in \mathfrak{K}_{p}^{\text {loc }}$ implies $X$ is a $\mathfrak{K}_{p}^{\text {loc }}$-topological space. On the other hand, $X$ is not a $\mathfrak{K}_{p}$-topological space (cf. [6. p. 40]). This serves as a counter-example of $\mathfrak{A}^{\text {sup }}$ topology $=\mathfrak{A}^{\text {inf }}$-topology and $\mathfrak{A}^{\text {sup }}$-topological spaces $=\mathfrak{A}^{\text {inf }}$-topological spaces, although we always have $\mathfrak{A}^{\text {rup_topology }}=\mathfrak{A}^{\text {inf }}$-topology and $\mathfrak{A}^{\text {rup_topological spaces }}=\mathfrak{A}^{\text {inf }}$-topological spaces. By the way, $X$ is both $\mathfrak{K}_{p}$-bornological and $\mathfrak{K}_{p}^{\text {loc }}$ - bornological, i.e., a co-Schwartz space but not a Schwartz space. 
Proposition 5.19. Let $\mathcal{P}$ be a generating topology on LCS's and $\mathfrak{A}=\mathcal{O}^{b}(\mathcal{P})$. The $\mathfrak{A}$-bornology coincides with the Grothendieck bornology generated by $\mathfrak{A}$ on each infracomplete LCS.

Proof. It follows from the easy fact $\mathfrak{A} \subseteq \mathfrak{A}_{\mathbb{B}}^{\text {lup }}$ and Theorems 5.13 and 5.15

Proposition 5.20. Let $\mathcal{P}$ be a generating topology on LCS's and $\mathfrak{A}=\mathcal{O}(\mathcal{P})$. Then the $\mathfrak{A}-$ bornology coincides with the $\left(\mathfrak{A}_{\mathbb{B}}^{\text {sur }}\right)^{\text {sup }}$-bornology on every infracomplete LCS.

Proof. Similar to Proposition 5.17

Example 5.21. Let $X=\mathbb{K}^{I}$ be the product space of card $(I)$ many $\mathbb{K}$ 's where the index set is uncountable. $X$ is infracomplete. Let $\mathcal{P}_{p c}$ be the generating topology defined by the precompact seminorms, i.e., $\mathcal{P}_{p c}=\mathcal{T}\left(\mathfrak{K}_{p}\right)$, where $\mathfrak{K}_{p}$ is the ideal of all precompact operators between LCS's (see Wong [30]). Then $\mathcal{O}^{b}\left(\mathcal{P}_{p c}\right)$ is the ideal $\mathfrak{K}_{p}^{b}$ of all quasi-Schwartz (= precompact-bounded, cf. Rankte [16]) operators between LCS's. $\mathcal{O}\left(\mathcal{P}_{p c}\right)$ is the ideal of those continuous operators between LCS's which are still continuous when the domain space $X$ equipped with the (coarser) precompact topology $\mathcal{P}_{p c}(X) . X$ is not a $\mathfrak{K}_{p}^{b}$-bornological space since otherwise (by Theorem 5.3) we would have the canonical embedding from $\mathbb{K}^{(I)}$ into $\mathbb{K}^{I}$ being quasi-Schwartz and this is not the case as shown in [11, p. 399]. $X$ is, however, an $\mathcal{O}\left(\mathcal{P}_{p c}\right)$-bornological space since all bounded sets in $X$ are precompact. This serves as a counter-example of $\mathfrak{A}^{\text {sup }}$-bornology $=$ $\mathfrak{A}^{\text {inf }}$-bornology and $\mathfrak{A}^{\text {sup }}$ - bornological spaces $=\mathfrak{A}^{\text {inf }}$-bornological spaces, although we always have $\mathfrak{A}^{\text {rup }- \text { bornology }}=\mathfrak{A}^{\text {inf }}$ - bornology on every infracomplete LCS. By the way, $X$ is both $\mathfrak{K}_{p}^{b}$-topological and $\mathcal{O}\left(\mathcal{P}_{p c}\right)$-topological, i.e., a Schwartz space but not a co-Schwartz space.

Remark 5.22. It may be interesting to study the $\mathfrak{A}^{\text {sup }}$-topology and the $\mathfrak{A}^{\text {sup }}$-bornology for an operator ideal $\mathfrak{A}$ on Banach spaces. Propositions [5.17 and 5.20] suggest the conjectures that $\mathcal{O}(\mathfrak{T}(\mathfrak{A}))=\left(\mathfrak{A}_{\mathbb{B}}^{\text {inj }}\right)^{\text {sup }}$ and $\mathcal{O}(\mathcal{B}(\mathfrak{A}))=\left(\mathfrak{A}_{\mathbb{B}}^{\text {sur }}\right)^{\text {sup }}$ where $\mathfrak{A}$ is an operator ideal on LCS's.

Theorem 5.23. Let $\mathfrak{A}$ be an operator ideal on LCS's, and X a LCS. Then

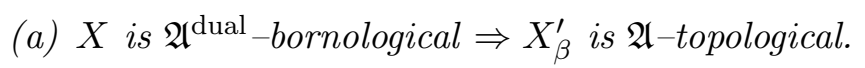

If, in addition, $X$ is infrabarrelled then

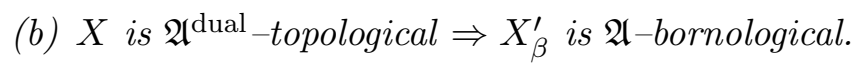

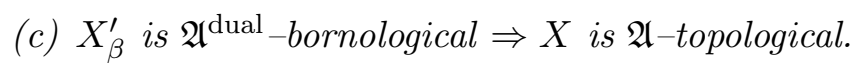

If, in addition to all above, $\mathfrak{A}$ is also injective then 


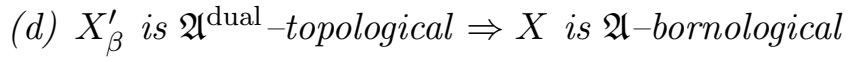

Proof. (a) Let $V$ be an absolutely convex, closed 0 -neighborhood in $X_{\beta}^{\prime}$. Then $V^{\circ}$ is bounded

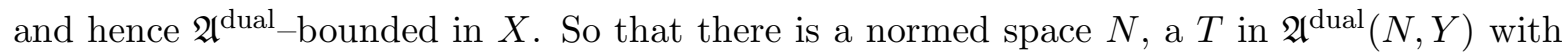
$T U_{N} \supseteq V^{\circ}$. Consequently, $\left(T U_{N}\right)^{\circ}=\left(T^{\prime}\right)^{-1} U_{N^{\prime}} \subseteq V^{\circ \circ}=V$ and $T^{\prime} \in \mathfrak{A}\left(X_{\beta}^{\prime}, N^{\prime}\right)$. It follows that $V$ is an $\mathfrak{A}$-neighborhood of 0 in $X_{\beta}^{\prime}$.

(b) Let $B$ be a bounded set in $X_{\beta}^{\prime}$. Then $B^{\circ}$ is a closed bornivorous barrel in $X$, and hence a 0 -neighborhood, and consequently an $\mathfrak{A}^{\text {dual }}$-neighborhood of 0 in $X$. Therefore there is a Banach space $F$, an $T$ in $\mathfrak{A}^{\text {dual }}(X, F)$ such that $B^{\circ} \supseteq T^{-1}\left(U_{F}\right)$. It follows $B \subseteq B^{\circ \circ} \subseteq T^{\prime} U_{F^{\prime}}$. Since $T^{\prime} \in \mathfrak{A}\left(F^{\prime}, X_{\beta}^{\prime}\right), B$ is $\mathfrak{A}$-bounded in $X_{\beta}^{\prime}$.

(c) Let $V$ be an absolutely convex, closed 0 -neighborhood in $X$. Then $V^{\circ}$ is bounded and hence $\mathfrak{A}^{\text {dual }}$-bounded in $X_{\beta}^{\prime}$. So that there is a normed space $N$ and a $T$ in $\mathfrak{A}^{\text {dual }}\left(N, X_{\beta}^{\prime}\right)$ such that $T U_{N} \supseteq V^{\circ}$ and thus $\left(T^{\prime}\right)^{-1} U_{N^{\prime}} \subseteq V^{\circ}$, where $V^{\circ}$ is the polar of $V^{\circ}$ in the strong bidual $X_{\beta \beta}^{\prime \prime}$ of $X$. It follows that $V^{\circ}$ is an $\mathfrak{A}$-neighborhood of 0 in $X_{\beta \beta}^{\prime \prime}$. Since the evaluation map $K_{X}: X \rightarrow X_{\beta \beta}^{\prime \prime}$ is continuous, $K_{X}^{-1}\left(V^{\bullet}\right)=V$ is an $\mathfrak{A}$-neighborhood of 0 in $X$ by $\left(\mathrm{GT}_{2}\right)$.

(d) Let $B$ be an absolutely convex bounded set in $X$. It suffices to check that $J_{B} \in$ $\mathfrak{A}^{\text {bsur }}(X(B), X)$. Note that $B^{\circ}$ is a neighborhood of 0 , and hence an $\mathfrak{A}^{\text {dual }}$-neighborhood of 0 in $X_{\beta}^{\prime}$. Hence there exist a Banach space $F$ and a $T$ in $\mathfrak{A}^{\text {dual }}\left(X_{\beta}^{\prime}, F\right)$ such that $B^{\circ} \supseteq T^{-1}\left(U_{F}\right)$. Hence $B^{\circ \bullet} \subseteq T^{\prime} U_{F^{\prime}}$. Since $T^{\prime} \in \mathfrak{A}\left(F^{\prime}, X_{\beta \beta}^{\prime \prime}\right), B^{\circ \bullet}$ is $\mathfrak{A}$-bounded in $X_{\beta \beta}^{\prime \prime}$ and thus $J_{B^{\circ} \bullet} \in$ $\mathfrak{A}^{\text {bsur }}\left(X_{\beta \beta}^{\prime \prime}\left(B^{\circ \bullet}\right), X_{\beta \beta}^{\prime \prime}\right)$. Now $K_{X} B \subseteq B^{\circ \bullet}$ ensures that there is a $K_{B}$ in $\mathcal{L}\left(X(B), X_{\beta \beta}^{\prime \prime}\left(B^{\circ \bullet}\right)\right)$ such that $J_{B} \bullet K_{B}=K_{X} J_{B}$. Hence $K_{X} J_{B} \in \mathfrak{A}^{\text {bsur }}\left(X(B), X_{\beta \beta}^{\prime \prime}\right)$ and it follows

$$
J_{B} \in\left(\mathfrak{A}^{\text {bsur }}\right)^{\text {inj }}(X(B), X)=\left(\mathfrak{A}^{\text {inj }}\right)^{\text {bsur }}(X(B), X)=\mathfrak{A}^{\text {bsur }}(X(B), X),
$$

by Proposition 3.2

Theorem 5.24. Let $\mathfrak{A}$ be a symmetric operator ideal (i.e., $\mathfrak{A} \subseteq \mathfrak{A}^{\text {dual }}$ ) on $L C S$ 's, and $X$ an infrabarrelled LCS. Then

(a) $X$ is $\mathfrak{A}$-topological $\Leftrightarrow X_{\beta}^{\prime}$ is $\mathfrak{A}$-bornological.

If, in addition, $\mathfrak{A}$ is injective, then

(b) $X$ is $\mathfrak{A}$-bornological $\Leftrightarrow X_{\beta}^{\prime}$ is $\mathfrak{A}$-topological.

Proof. A consequence of Theorem 5.23. 
Theorem 5.25. Let $\mathcal{P}$ be a generating topology on LCS's and $X$ be an infrabarrelled LCS. Then

(a) $X$ is $\mathcal{O}^{b}\left(\mathcal{P}^{\circ}\right)$-topological $\Rightarrow X_{\beta}^{\prime}$ is $\mathcal{O}^{b}(\mathcal{P})$-bornological.

(b) $X$ is $\mathcal{O}^{b}\left(\mathcal{P}^{\circ}\right)$-bornological $\Rightarrow X_{\beta}^{\prime}$ is $\mathcal{O}^{b}(\mathcal{P})$-topological.

(c) $X_{\beta}^{\prime}$ is $\mathcal{O}^{b}\left(\mathcal{P}^{\circ}\right)$-topological $\Rightarrow X$ is $\mathcal{O}^{b}(\mathcal{P})$-bornological.

(d) $X_{\beta}^{\prime}$ is $\mathcal{O}^{b}\left(\mathcal{P}^{\circ}\right)$-bornological $\Rightarrow X$ is $\mathcal{O}^{b}(\mathcal{P})$-topological.

In case $\mathcal{O}(\mathcal{P})$ is symmetric or $\mathcal{P}$ has the subspace property, all above implications become equivalences.

Proof. We prove (c) only and all others are similar. Suppose $X_{\beta}^{\prime}$ is $\mathcal{O}^{b}\left(\mathcal{P}^{\circ}\right)$-topological. By

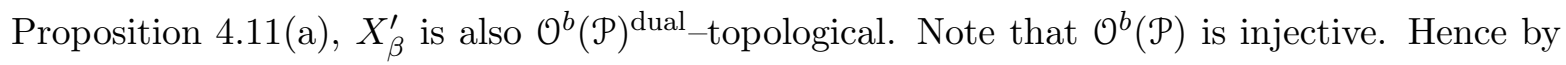
Theorem $5.23(\mathrm{~d}), X$ is $\mathcal{O}^{b}(\mathcal{P})$-bornological. In case $\mathcal{O}(\mathcal{P})$ is symmetric or $\mathcal{P}$ has the subspace property, if $X$ is $\mathcal{O}^{b}(\mathcal{P})$-bornological, $X$ is also $\mathcal{O}^{b}\left(\mathcal{P}^{\circ}\right)^{\text {dual }}$ - bornological by Proposition 4.11(b) . By Theorem 5.23(a), $X_{\beta}^{\prime}$ is $\mathcal{O}^{b}\left(\mathcal{P}^{\circ}\right)$-topological, as asserted.

Example 5.26. Let $\mathcal{P}_{\sigma}$ be the generating system of $\sigma\left(X, X^{\prime}\right)$-topology on each LCS $X$. $\mathcal{P}_{\sigma}^{\circ}(X)$ is thus the convex bornology $\mathcal{M}(X)$ consisting of those bounded subsets $B$ of $X$ whose polars $B^{\circ}$ are $\sigma\left(X_{\beta}^{\prime}, X_{\beta \beta}^{\prime \prime}\right)$-neighborhoods of 0 in $X_{\beta}^{\prime}$. Now both $\mathcal{O}^{b}\left(\mathcal{P}_{\sigma}\right)$ and $\mathcal{O}^{b}\left(\mathcal{P}_{\sigma}^{\circ}\right)$ define the ideal $\mathfrak{F}$ of continuous operators of finite rank. Moreover, $\mathcal{P}_{\sigma}$ has the subspace property. Theorem 5.25 applies and says that for an infrabarrelled LCS $X$, we have

(a) $X$ is $\mathfrak{F}$-topological if and only if $X_{\beta}^{\prime}$ is $\mathfrak{F}$-bornological;

(b) $X$ is $\mathfrak{F}$-bornological if and only if $X_{\beta}^{\prime}$ is $\mathfrak{F}$-topological.

Unlike the case of Banach spaces, an $\mathfrak{F}$-topological or $\mathfrak{F}$-bornological LCS need not be of finite dimension. For examples, the LCS $\mathbb{K}^{I}$ is $\mathfrak{F}$-topological and the LCS $\mathbb{K}^{(I)}$ is $\mathfrak{F}$-bornological. This is because the weak topology $\sigma\left(\mathbb{K}^{I}, \mathbb{K}^{(I)}\right)$ of $\mathbb{K}^{I}$ coincides with the product topology of $\mathbb{K}^{I}$ and every bounded set in $\mathbb{K}^{(I)}$ is of finite dimension. Here the index set $I$ is arbitrary.

\section{Examples And ApPlichtions}

This last section is devoted to examples and applications, showing the powerful techniques developed in the previous sections. Many other elegant applications of the theory of Grothendieck spaces and co-Grothendieck spaces can be found, for example, in [8, 11], [12, 14], 15. The concepts of $\mathfrak{A}$-topological spaces and $\mathfrak{A}$-bornological spaces are also welldeveloped in the context in [6], 14, [16, 29, 32, and, in particular, 30]. 


\subsection{Schwartz spaces and co-Schwartz spaces.}

Definition 6.1 (see, e.g., [30, p. 14]). A continuous seminorm $p$ on a LCS $X$ is said to be precompact if there exists a $\left(\lambda_{n}\right)$ in $c_{0}$ and an equicontinuous sequence $\left\{x_{n}^{\prime}\right\}$ in $X^{\prime}$ such that

$$
p(x) \leq \sup \left\{\left|\lambda_{n}\left\langle x, x_{n}^{\prime}\right\rangle\right|: n \geq 1\right\}, \quad \forall x \in X .
$$

Denote by $\mathcal{P}_{p c}(X)$ the locally convex (Hausdorff) topology on $X$ defined by all precompact seminorms on $X$. It is easy to see that $\mathcal{P}_{p c}=\left\{\mathcal{P}_{p c}(X): X\right.$ is a $\left.L C S\right\}$ is a generating topology. It is a classical result (cf. [16] or [30]) that $p$ is a precompact seminorm on a LCS $X$ if and only if the canonical map $Q_{p}: X \rightarrow X_{p}$ is precompact. Then, $\mathfrak{K}_{p}=\mathcal{O}\left(\mathcal{P}_{p c}\right)$ is the ideal of all precompact operators, and $\mathfrak{K}_{p}^{b}=\mathcal{O}^{b}\left(\mathcal{T}\left(\mathfrak{K}_{p}\right)\right)$ is the ideal of all quasi-Schwartz (i.e., precompactbounded) operators between LCS's.

Definition 6.2. A LCS $X$ is said to be a Schwartz space if every continuous seminorm $p$ on $X$ is precompact.

We provide a new proof of the following classical result.

Theorem 6.3 (see [30, pp. 17 and 26]). Let $X$ be a LCS. The following are all equivalent.

(a) $X$ is a Schwartz space.

(b) For each continuous seminorm $p$ on $X$ there is a continuous seminorm $q$ on $X$ such that $p \leq q$ and the canonical map $Q_{p q}$ belongs to $\mathfrak{K}_{p}\left(X_{q}, X_{p}\right)$.

(c) $Q_{p} \in \mathfrak{K}_{p}\left(X, X_{p}\right)$ for every continuous seminorm $p$ on $X$.

(d) For any 0-neighborhood $U$ in $X$ there exists a 0 -neighborhood $V$ in $X$ such that $V \subseteq U$ and the canonical map from $X^{\prime}\left(U^{\circ}\right)$ into $X^{\prime}\left(V^{\circ}\right)$ is precompact.

(e) $\mathcal{L}(X, N)=\mathfrak{K}_{p}(X, N)$ for every normed (or Banach) space $N$.

(f) $\mathfrak{K}_{p}^{b}(X, Y)=\mathcal{L}^{b}(X, Y)$ for every LCS $Y$.

(g) $\mathfrak{K}_{p}^{b}(X, Y)=\mathfrak{K}_{p}(X, Y)$ for every LCS $Y$.

(h) $\mathcal{L}(X, N)=\mathfrak{K}_{p}^{b}(X, N)$ for every normed (or Banach) space $N$.

(i) $X$ is a $\mathfrak{K}_{p}$-topological space.

Proof. (a) $\Leftrightarrow(\mathrm{c}) \Leftrightarrow(\mathrm{e}) \Leftrightarrow(\mathrm{i})$ are due to Theorem 5.1 and the injectivity of $\mathfrak{K}_{p}$. (a) $\Leftrightarrow(\mathrm{b})$ because $\mathfrak{K}_{p}=\mathcal{O}^{b}\left(\mathcal{M}_{p c}\right)$ where $\mathcal{M}_{p c}$ is the generating bornology of precompact sets and Proposition 5.16 applies. $(\mathrm{b}) \Leftrightarrow(\mathrm{d})$ follows from the complete symmetry of the restriction $\left(\mathfrak{K}_{p}\right)_{\mathbb{B}}$ of $\mathfrak{K}_{p}$ to Banach spaces, i.e., $\left(\mathfrak{K}_{p}\right)_{\mathbb{B}}^{\text {dual }}=\left(\mathfrak{K}_{p}\right)_{\mathbb{B}} .(\mathrm{a}) \Leftrightarrow(\mathrm{f}) \Leftrightarrow(\mathrm{h})$ are consequences of Theorem $5.1(\mathrm{i}) \Rightarrow(\mathrm{g})$ is contained in Proposition 4.7 and Theorem [5.5. Finally, for $(\mathrm{g}) \Rightarrow(\mathrm{h})$, denote by $N_{\sigma}$ the 
$\operatorname{LCS}\left(N, \sigma\left(N, N^{\prime}\right)\right)$. For every $T$ in $\mathcal{L}(X, N), T \in \mathcal{L}\left(X, N_{\sigma}\right)=\mathfrak{K}_{p}\left(X, N_{\sigma}\right)$. Hence, by (g), $T \in \mathfrak{K}_{p}^{b}\left(X, N_{\sigma}\right)=\mathfrak{K}_{p}^{b}(X, N)$ since $N$ and $N_{\sigma}$ carry the same (von Neumann) bornology.

Definition 6.4. A LCS $Y$ is said to be a co-Schwartz space if its strong dual $Y_{\beta}^{\prime}$ is a Schwartz space.

Theorem 6.5. Let $Y$ be a LCS. Consider the following statements.

(a) $Y$ is a co-Schwartz space.

(b) For each bounded disk $B$ in $Y$ there is a bounded disk $A$ in $Y$ with $B \subseteq A$ such that the canonical map $J_{A B}$ from $Y(B)$ into $Y(A)$ belongs to $\mathfrak{K}_{p}(Y(B), Y(A))$.

(c) $J_{B} \in \mathfrak{K}_{p}(Y(B), Y)$ for each bounded disk $B$ in $Y$.

(d) $\mathcal{L}(N, Y)=\mathfrak{K}_{p}(N, Y)$ for every normed space $N$.

(e) $\mathcal{L}^{b}(X, Y)=\mathfrak{K}_{p}(X, Y)$ for every $L C S X$.

(f) $Y$ is a $\mathfrak{K}_{p}$-bornological space.

We have $(a) \Leftrightarrow(b) \Rightarrow(c) \Leftrightarrow(d) \Leftrightarrow(e) \Leftrightarrow(f)$.

Proof. (a) $\Leftrightarrow$ (b) follows from the equivalence (a) $\Leftrightarrow(\mathrm{b})$ in the last theorem and the complete symmetry of $\left(\mathfrak{K}_{p}\right)_{\mathbb{B}}$. (c) $\Leftrightarrow(\mathrm{d}) \Leftrightarrow(\mathrm{e}) \Leftrightarrow(\mathrm{f})$ are just examples of Theorem [5.3. (b) $\Rightarrow(\mathrm{c})$ is trivial. Finally, the LCS $\mathbb{K}^{I}$, where the index set $I$ is uncountable, furnishes a counter-example of the missing implication.

Proposition 6.6. Let $X$ be an infrabarrelled LCS. Then $X$ is a Schwartz space (resp. coSchwartz space) if and only if $X_{\beta}^{\prime}$ is a co-Schwartz space (resp. Schwartz space) if and only if $X_{\beta \beta}^{\prime \prime}$ is a Schwartz space (resp. co-Schwartz space).

Proof. Repeat applying Theorems 6.3 and 6.5] and the complete symmetry of $\left(\mathfrak{K}_{p}\right)_{\mathbb{B}}$.

Remark 6.7. Besides the ideals of precompact operators and quasi-Schwartz operators, one can also employ the ideal $\mathcal{L}_{\text {im }}$ of limit operators to define Schwartz spaces and co-Schwartz spaces. See 12] for some other internal characterization of Schwartz spaces due to the introduction of $\mathcal{L}_{\mathrm{im}}$.

Similar to Schwartz space we can relate infra-Schwartz spaces to the ideal $\mathfrak{W}$ of weakly compact operators between LCS's. Incidentally, readers should have no difficulty to figure out that $\mathfrak{K}_{p}$-bornological spaces are, in fact, semi-Montel spaces and $\mathfrak{W}$-bornological spaces are exactly semi-reflexive spaces. We leave these to the interested readers and refer them to [6] for more information about the classical theory of these spaces. 


\subsection{Nuclear spaces and co-nuclear spaces.}

Definition 6.8. A continuous seminorm $p$ on a LCS $X$ is called an absolutely summing seminorm (= prenuclear seminorm in [30]) if there exists a $\sigma\left(X^{\prime}, X\right)$-closed equicontinuous subset $B$ of $X^{\prime}$ and a positive Radon measure $\mu$ on $B$ such that

$$
p(x) \leq \int_{B}\left|\left\langle x, x^{\prime}\right\rangle\right| d \mu\left(x^{\prime}\right), \quad \forall x \in X
$$

Let $\mathcal{P}_{\text {as }}(X)$ be the locally convex (Hausdorff) topology on $X$ generated by the family of all absolutely summing seminorms on $X$. It is easy to see that the system $\mathcal{P}_{\text {as }}=\left\{\mathcal{P}_{\text {as }}(X): X\right.$ a $L C S\}$ is a generating topology. A continuous operator $T$ from a LCS $X$ into a LCS $Y$ is said to be absolutely summing if $T \in \mathcal{O}\left(\mathcal{P}_{\text {as }}\right)(X, Y)=\mathcal{L}\left(X_{\mathcal{P}_{\text {as }}}, Y\right)$. In case $X$ and $Y$ are Banach spaces, $T$ is absolutely summing if and only if $T$ sends every weakly summable series in $X$ to an absolutely summable series in $Y$. Denote by $\mathfrak{P}=\mathcal{O}\left(\mathcal{P}_{\text {as }}\right)$ the injective ideal of all absolutely summing operators between LCS's, and by $\mathfrak{P}^{b}=\mathcal{O}^{b}\left(\mathcal{P}_{\text {as }}\right)$ the injective ideal of prenuclear-bounded operators [30].

A continuous operator $T$ from a LCS $X$ into a LCS $Y$ is said to be nuclear if there exist a $\left(\lambda_{n}\right)$ in $l_{1}$, an equicontinuous sequence $\left\{a_{n}\right\}$ in $X^{\prime}$ and a sequence $\left\{y_{n}\right\}$ contained in an infracomplete bounded disk $B$ in $Y$ such that $T=\Sigma_{n} \lambda_{n} a_{n} \otimes y_{n}$, i.e., $T x=\Sigma_{n} \lambda_{n} a_{n}(x) y_{n}$ for each $x$ in $X$. Denote by $\mathfrak{N}$ the ideal of all nuclear operators between LCS's. Note that $\mathfrak{N}$ is

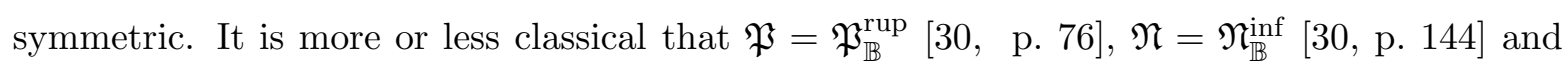
$\mathfrak{P}_{\mathbb{B}}^{3} \subset \mathfrak{N}_{\mathbb{B}} \subset \mathfrak{P}_{\mathbb{B}}\left[30\right.$ p. 145] (in fact, we have $\mathfrak{P}_{\mathbb{B}}^{2} \subset \mathfrak{N}_{\mathbb{B}},\left(\mathfrak{N}_{\mathbb{B}}^{\text {inj }}\right)^{2} \subset \mathfrak{N}_{\mathbb{B}}$, cf. [14]).

Definition 6.9. A LCS $X$ is said to be nuclear if every continuous seminorm $p$ on $X$ is absolutely summing. A LCS $Y$ is said to be co-nuclear if its strong dual $Y_{\beta}^{\prime}$ is nuclear.

We provide a new proof of the following classical result.

Theorem 6.10 (see [30, pp. 149 and 157]). Let X be a LCS. The following are all equivalent.

(a) $X$ is a nuclear space.

(b) $Q_{p} \in \mathfrak{P}\left(X, X_{p}\right)$ for every continuous seminorm $p$ on $X$.

(c) For each continuous seminorm $p$ on $X$ there exists a continuous seminorm $q$ on $X$ with $p \leq q$ such that the canonical map $Q_{p q} \in \mathfrak{P}\left(X_{q}, X_{p}\right)$.

(d) $i d_{X} \in \mathfrak{P}(X, X)$.

(e) $\mathfrak{P}(X, Y)=\mathcal{L}(X, Y)$ for every $L C S Y$.

(f) $\mathfrak{P}(X, N)=\mathcal{L}(X, N)$ for every normed space $N$. 
(g) $\widetilde{Q}_{p} \in \mathfrak{N}\left(X, \widetilde{X}_{p}\right)$ for every continuous seminorm $p$ on $X$.

(h) $\mathfrak{N}(X, F)=\mathcal{L}(X, F)$ for every Banach space $F$.

(i) For each continuous seminorm $p$ on $X$ there exists a continuous seminorm $q$ on $X$ with $p \leq q$ such that the canonical map $\widetilde{Q}_{p q} \in \mathfrak{N}\left(\widetilde{X}_{q}, \widetilde{X}_{p}\right)$.

(j) For each 0-neighborhood $V$ in $X$ there is a 0 -neighborhood $U$ in $X$ with $U \subseteq V$ such that the canonical map $X^{\prime}\left(V^{\circ}\right) \rightarrow X^{\prime}\left(U^{\circ}\right)$ is nuclear.

(k) $\mathfrak{P}^{b}(X, Y)=\mathcal{L}^{b}(X, Y)$ for every LCS $Y$.

(l) $\mathcal{L}^{b}(X, Y) \subseteq \mathfrak{P}(X, Y)$ for every $L C S Y$.

(m) $\mathfrak{K}_{p}(X, Y) \subseteq \mathfrak{P}^{b}(X, Y)$ for every LCS $Y$.

(n) $\mathcal{L}^{b}(X, Y) \cap \mathfrak{P}(X, Y)=\mathfrak{P}^{b}(X, Y)$ for every LCS $Y$.

(o) $X$ is a $\mathfrak{P}$-topological space.

(p) $X$ is a $\mathfrak{N}$-topological space.

Proof. (a) $\Leftrightarrow$ (b) $\Leftrightarrow$ (d) $\Leftrightarrow$ (e) $\Leftrightarrow$ (f) $\Leftrightarrow$ (l) $\Leftrightarrow$ (o) $\Leftrightarrow$ (p) are due to Theorem 5.1. Since $\mathfrak{P}=\mathfrak{P}_{\mathbb{B}}^{\text {rup }}$, we have $(\mathrm{a}) \Leftrightarrow(\mathrm{c})$ by Theorem 5.15, (a) $\Leftrightarrow(\mathrm{n}) \Leftrightarrow(\mathrm{k})$ are due to Proposition 4.7 and Theorem [5.5. $(\mathrm{k}) \Rightarrow(\mathrm{m})$ is obvious. To prove $(\mathrm{m}) \Rightarrow(\mathrm{k})$ we employ the same trick as in Theorem 6.3. (c) $\Leftrightarrow$ (i) follows from the fact that $\mathfrak{P}^{3} \subset \mathfrak{N} \subset \mathfrak{P}$. (i) $\Rightarrow(\mathrm{g}) \Rightarrow$ (h) are trivial. $(\mathrm{h}) \Rightarrow(\mathrm{l})$ because $\mathfrak{N} \subseteq \mathfrak{P}$ and $\mathfrak{P}$ is injective. (i) $\Rightarrow(\mathrm{j})$ is ensured by the symmetry of $\mathfrak{N}_{\mathbb{B}}$.

Finally, we prove $(\mathrm{j}) \Rightarrow(\mathrm{i})$. Let $V_{p}=\{x \in X: p(x) \leq 1\}$ be the 0 -neighborhood associated to a continuous seminorm $p$ on $X$. By $(\mathrm{j})$, there is a continuous seminorm $q$ on $X$ such that $V_{q} \subseteq V_{p}$ (i.e., $\left.p \leq q\right)$ and $\widetilde{Q}_{p q}^{\prime}: X^{\prime}\left(V_{p}^{\circ}\right) \rightarrow X^{\prime}\left(V_{q}^{\circ}\right)$ is nuclear. By the symmetry of $\mathfrak{N}_{\mathbb{B}}$, $\widetilde{Q}_{p q}^{\prime \prime}:\left(X^{\prime}\left(V_{q}^{\circ}\right)\right)^{\prime} \rightarrow\left(X^{\prime}\left(V_{p}^{\circ}\right)\right)^{\prime}$ is nuclear, too. Hence $\widetilde{Q}_{p q}^{\prime \prime}$ is absolutely summing. Now $\left(X^{\prime}\left(V_{q}^{\circ}\right)\right)^{\prime}$ and $\left(X^{\prime}\left(V_{p}^{\circ}\right)\right)^{\prime}$ are isometrically isomorphic to $X_{q}^{\prime \prime}$ and $X_{p}^{\prime \prime}$, respectively. By the injectivity of $\mathfrak{P}$, $\widetilde{Q}_{p q}$ is absolutely summing. Repeating the same argument, we shall have continuous seminorms $q_{1}$ and $q_{2}$ on $X$ such that $q \leq q_{1} \leq q_{2}$ and $\widetilde{Q}_{q q_{1}}$ and $\widetilde{Q}_{q_{1} q_{2}}$ are both absolutely summing. Now $p \leq q_{2}$ and $\widetilde{Q}_{p q_{2}}=\widetilde{Q}_{p q} \widetilde{Q}_{q q_{1}} \widetilde{Q}_{q_{1} q_{2}} \in \mathfrak{P}_{\mathbb{B}}^{3} \subseteq \mathfrak{N}_{\mathbb{B}}$, and we are done.

Remark 6.11. There are concepts of quasi-nuclear-seminorms, quasi-nuclear operators and quasi-nuclear-bounded operators, cf. 30 . They can be used to define nuclear spaces like $\mathfrak{P}$ and $\mathfrak{N}$. However, they are simply, respectively, the $\mathfrak{N}$-seminorms, $\mathfrak{N}^{\text {inj }}$-operators and $\left(\mathcal{T}\left(\mathfrak{N}^{\text {inj }}\right)\right)^{b_{-}}$ operators. Using the same kind of argument in Theorem 6.10 one can easily prepare a longer list of equivalences. We leave this to the interested readers.

Theorem 6.12. Let $Y$ be an infrabarrelled LCS. The following are all equivalent.

(a) $Y$ is a co-nuclear space. 
(b) For each bounded disk $B$ in $Y$ there is a bounded disk $A$ in $Y$ with $B \subseteq A$ such that the canonical map $J_{A B}$ from $Y(B)$ into $Y(A)$ is nuclear.

(c) $J_{B} \in \mathfrak{N}(Y(B), Y)$ for every bounded disk $B$ in $Y$.

(d) $\mathcal{L}(N, Y)=\mathfrak{N}(N, Y)$ for every normed space $N$.

(e) $\mathcal{L}^{b}(X, Y) \subseteq \mathfrak{N}(X, Y)$ for every $L C S X$.

(f) $Y$ is an $\mathfrak{N}$-bornological space.

Proof. Assume first that $Y$ is co-nuclear and $B$ is a bounded disk in $Y$. Then $B^{\circ}$ is a $0-$ neighborhood in $Y_{\beta}^{\prime}$. Hence there is a bounded disk $A$ in $Y$ with $B \subseteq A$ such that the canonical map $Y^{\prime \prime}\left(B^{\circ}\right) \rightarrow Y^{\prime \prime}\left(A^{\bullet \bullet}\right)$ is absolutely summing. Since $\mathfrak{P}$ is injective, the canonical map $J_{A B}$ from $Y(B)$ into $Y(A)$ is also absolutely summing. Do this twice more and we shall get (a) $\Rightarrow$ (b) since $\mathfrak{P}_{\mathbb{B}}^{3} \subseteq \mathfrak{N}_{\mathbb{B}}$. (b) $\Rightarrow$ (c), (d), (e) and each one of them $\Rightarrow$ (f) are straightforward. We consider ( $\mathrm{f}) \Rightarrow$ (a). Note that $\mathfrak{N}$ is symmetric. Now Theorem $[5.23$ (a) gives the desired conclusion.

Proposition 6.13. Let $X$ be an infrabarrelled LCS. Then $X$ is a nuclear space (resp. conuclear space) if and only if $X_{\beta}^{\prime}$ is a co-nuclear space (resp. nuclear space) if and only if $X_{\beta \beta}^{\prime \prime}$ is a nuclear space (resp. co-nuclear space).

Proof. In view of Theorem 5.25] it suffices to mention that the generating system $\mathcal{P}_{\text {as }}$ of absolutely summing topology has the subspace property. As a result, an infrabarrelled LCS $X$ is nuclear if and only if $X_{\beta \beta}^{\prime \prime}$ is nuclear. The other implications follow from this.

Since $\mathfrak{N} \subset \mathfrak{K}_{p}$ we have the well-known

Proposition 6.14. All nuclear (resp. co-nuclear) spaces are Schwartz (resp. co-Schwartz) spaces.

6.3. Permanence properties. We collect some results from [11] about the permanence properties of Grothendieck spaces and co-Grothendieck spaces.

Theorem 6.15 (11, Junek]). Let $\mathfrak{A}$ be an operator ideal on Banach spaces.

(a) Any product of $\operatorname{Groth}(\mathfrak{A})$-spaces is a $\operatorname{Groth}(\mathfrak{A})$-space.

(b) Any locally convex direct sum of co-Groth( $\mathfrak{A})$-spaces is a co-Groth( $\mathfrak{A})$-space.

(c) If $\mathfrak{A}$ is equivalent to some injective ideal then any subspace of a $\operatorname{Groth}(\mathfrak{A})$-space is a $\operatorname{Groth}(\mathfrak{A})$-space. 
(d) If $\mathfrak{A}$ is equivalent to some surjective ideal then any quotient space of a co-Groth( $\mathfrak{A})$-space is a co- $\operatorname{Groth}(\mathfrak{A})$-space.

(e) If $\mathfrak{A}$ is injective then any projective limit of $\operatorname{Groth}(\mathfrak{A})$-spaces is a $\operatorname{Groth}(\mathfrak{A})$-space.

(f) If $\mathfrak{A}$ is injective then any subspace of a co-Groth( $\mathfrak{A})$-space is a co-Groth( $\mathfrak{A})$-space.

If one applies them together with other results in the earlier parts of this paper to Schwartz spaces, infra-Schwartz spaces, nuclear spaces, and their "co-spaces", one can obtain a long list of permanence properties of these spaces, cf. [8] or [11].

6.4. Other applications. Along the same line of reasoning in this paper one can develop similar applications of operator ideals to the theory of tensor products, partially ordered locally convex spaces and $C^{*}$-algebras.

It is of no doubt that the initial idea of operator ideals comes from tensor products. In 13 , p. 49], Michor suggested a method to construct a tensor norm $\mathfrak{A}^{\otimes}$ associated to each operator ideal $\mathfrak{A}$ on Banach spaces (see [15] for details about quasi-normed ideal). See also [30] and those famous works of A. Grothendieck and R. Schatten.

Let $E$ and $F$ be Banach spaces and $\mathfrak{A}$ be an operator ideal on Banach spaces with ideal norm $\alpha$. Define $\|\cdot\|_{\mathfrak{A} \otimes}$ on $E \otimes F$ by

$$
\left\|\sum x_{i} \otimes y_{i}\right\|_{\mathfrak{A}^{\otimes}}=\sup \left\{\left|\sum\left\langle y_{i}, T x_{i}\right\rangle\right|: T \in \mathfrak{A}\left(E, F^{\prime}\right), \quad \alpha(T) \leq 1\right\} .
$$

$\|\cdot\|_{\mathfrak{A} \otimes}$ turns out to be a reasonable cross norm. We denote by $E \otimes_{\mathfrak{A}} F$ the $\mathfrak{A}$-tensor product of $E$ and $F$, that is, the completion of $E \otimes F$ under $\|\cdot\|_{\mathfrak{A} \otimes}$. Y. C. Wong [30, p. 279] showed that if $\mathfrak{A}$ is the normed ideal $(\mathfrak{P}, P)$ of all absolutely summing operators between Banach spaces, we would have $\left(E \otimes_{\mathfrak{P}} F\right)^{\prime} \cong\left(\mathfrak{P}\left(E, F^{\prime}\right), P\right)$.

In general, let $\mathfrak{A}$ be an operator ideal on LCS's. We can define a tensor product topology associated to $\mathfrak{A}$ by a family of $\mathfrak{A}$-bilinear forms. A continuous bilinear form $b$ on $X \times Y$ is said to be an $\mathfrak{A}$-bilinear form, if there is a $T$ in $\mathfrak{A}\left(X, Y_{\beta}^{\prime}\right)$ such that $b(x, y)=\langle y, T x\rangle$. We write $b=b_{T}$ in this case. Detailed properties of $b_{T}$ can be found in [30. See also [7] for other comments. If $\mathfrak{A}$ is equipped with some locally convex topology (see [11]) then we can define similar seminorms like the one as $\|\cdot\|_{\mathfrak{A} \otimes}$. It might be interesting to investigate this kind of theory.

There is also an established theory of ideal topologies on partially ordered locally convex spaces. We give only one example here and refer interested readers to [29]. Let $\left(X, X_{+}, \mathcal{T}\right)$ be a locally solid space. A continuous seminorm $p$ on $X$ is said to be a $(P L)$-seminorm if 
there exists a positive $f$ in $X^{\prime}$ such that $p(x) \leq \sup \{g(x):-f \leq g \leq f\}, \forall x \in X$. It turns out that a continuous seminorm $p$ on a locally solid space $X$ is a $(P L)$-seminorm if and only if $Q_{p}$ is a cone-absolutely summing operator from $X$ onto $X_{p}$. Moreover, we have a list of characterizations of $\mathcal{T}$ to be the topology of uniform convergence on all order intervals as those appeared in Theorems 6.3 and 6.10 (see [29, p. 136]). We would like to mention that in the case of partially ordered locally convex spaces, or Banach lattices, the correct concept of operator ideals may be the so-called operator modules. For more information about operator modules, see Schwarz [19].

Finally, we finish this paper with a result of Jarchow [10]. Let $H$ be a Hilbert space and $A$ be a $C^{*}$-subalgebra of $B(H)$.

Proposition 6.16 (10, Jarchow]). The $\mathfrak{W}$-topology of A, i.e., the ideal topology on A generated by weakly compact operators, is the finest locally convex topology on A which coincides with the strong* (i.e., the double strong) operator topology on bounded subsets of $A$. The completion of $A$ under this topology is $\left(A^{* *}, \tau\left(A^{* *}, A^{*}\right)\right)$.

\section{REFERENCES}

[1] J. Conradie and G. West, "Topological and bornological characterisations of ideals in von Neumann algebras: II", Integral equations and operator theory, Integral Equations Operator Theory, 23 (1995), no. 1, 49-60.

[2] A. Defant and K. Floret, Tensor norms and operator ideal, Math. Studies 176, North-Holland, Amsterdam, 1993.

[3] L. Franco and C. Piñeiro, "The injective hull of an operator ideal on locally convex spaces", Manuscripa Math., 38 (1982), 333-341.

[4] A. Grothendieck, "Rèsumé de la theórie métrique des produits tensoriels topologiques", Bol. Soc. Mat. São Paulo, 8 (1956), 1-79.

[5] H. Hogbe-Nlend, Bornologies and functional analysis, Math. Studies 26, North-Holland, Amsterdam, 1977.

[6] _ Nuclear and co-nuclear spaces, Math. Studies 52, North-Holland, Amsterdam, 1981.

[7] G. J. O. Jameson, Summing and nuclear norms in Banach space theory, London Math. Soc. Students Text 8, Cambridge University Press, Cambridge, 1987.

[8] H. Jarchow, Locally Convex Spaces, Teubner, Stuttgart, 1981.

[9] _ _ "On certain locally convex topologies on Banach spaces", in Functional Analysis: Surveys and Recent Results, III, K. D. Bierstedt and B. Fuchsteiner (eds.), North-Holland, Amsterdam, 1984, 79-93.

[10] , "On weakly compact operators on $C^{*}$-algebras", Math. Ann., 273 (1986), 341-343.

[11] H. Junek, Locally convex spaces and operator ideals, Teubner-texte zur Math., Band 56, Teubner, Leipzig, 1983.

[12] M. Lindstorm, "A characterization of Schwartz spaces", Math. Z., 198 (1988), 423-430.

[13] P. Michor, Functors and categories of Banach spaces, Lecture Notes in Mathematics 651, Springer-Verlag, Berlin-Heidelberg-New York, 1978.

[14] A. Pietsch, Nuclear Locally Convex Spaces, Springer-Verlag, Berlin-Heidelberg-New York, 1972.

[15] — Operator Ideals, North-Holland, Amsterdam, 1980.

[16] D. Randtke, "Characterizations of precompact maps, Schwartz spaces and nuclear spaces", Trans. Amer. Math. Soc., 165 (1972), 87-101.

[17] H. H. Schaefer, Topological Vector Spaces, Springer-Verlag, Berlin-Heidelberg-New York, 1971.

[18] R. Schatten, Norm ideals of completely continuous operators, Ergebn. Math. Grenzgeb. 27, Springer, 1960.

[19] H.-U. Schwarz, Banach lattices and operators, Teubner-texte zur Mathematik, Band 71, Teubner, Leipzig, 1984. 
[20] I. Stephani, "Injektive operatorenideale über der gesamtheit aller Banachräume und ihre topologische erzeugung", Studia Math., 38 (1970), 105-124.

[21] _ "Surjektive operatorenideale über der gesamtheit aller Banachräume", Wiss. Z. FSU Jena, MathNat. R. Jg., 21 (1972), 187-216.

[22] _ "Surjektive operatorenideale über der gesamtheit aller Banachräume und ihre Erzeugung", Beiträge Analysis, . 5 (1973), 75-89.

[23] _ "Generating system of sets and quotients of surjective operator ideals", Math. Nachr., 99 (1980), $13-27$.

[24] _ "Generating topologies and quotients of injective operator ideals", in "Banach Space Theory and Its Application (proceedings, Bucharest 1981)", Lecture Notes in Math., 991, Springer-Verlag, BerlinHeidelberg-New York, 1983, 239-255.

[25] G. West, "Topological and bornological characterisations of ideals in von Neumann algebras: I", Integral Equations Operator Theory, 22 (1995), no. 3, 352-359.

[26] N.-C. Wong, "Topologies and bornologies determined by operator ideals, II", Studia Math. 111(2) (1994), $153-162$.

[27] _ "Yau-Chuen Wong", Southeast Asian Bull. Math., 20 no. 1 (1996), 1-3.

[28] _ and Y.-C. Wong, "The bornologically surjective hull of an operator ideal on locally convex spaces", Math. Narch., 160 (1993), 265-275.

[29] Y.-C. Wong, The topology of uniform convergence on order-bounded sets, Lecture Notes in Mathematics 531, Springer-Verlag, Berlin-Heidelberg-New York, 1976.

[30] Schwartz spaces, nuclear spaces and tensor products, Lecture Notes in Mathematics 726, SpringerVerlag, Berlin-Heidelberg-New York, 1979.

[31] — Introductory theory of topological vector spaces, Pure and Applied Mathematics, 167, Marcel Dekker, Inc., New York-Basel-Hong Kong, 1992.

[32] and N.-C. Wong, "Topologies and bornologies determined by operator ideals", Math. Ann., 282 (1988), 587-614.

Department of Applied Mathematics, National Sun Yat-sen University, and National Center for Theoretical Sciences, Kaohsiung, 80424, Taiwan, R.O.C.

E-mail address: wong@math.nsysu.edu.tw 